\title{
An Indicator of the Multiple Equilibria Regime of the Atlantic Meridional Overturning Circulation
}

\author{
Selma E. Huisman, Matthiss den Toom, and Henk A. Dijkstra \\ Institute for Marine and Atmospheric Research, Department of Physics and Astronomy, Utrecht University, \\ Utrecht, Netherlands \\ SYBREN DRIJFHOUT \\ Royal Netherlands Meteorological Institute, De Bilt, Netherlands
}

(Manuscript received 7 January 2009, in final form 4 October 2009)

\begin{abstract}
Recent model results have suggested that there may be a scalar indicator $\Sigma$ monitoring whether the Atlantic meridional overturning circulation (MOC) is in a multiple equilibrium regime. The quantity $\Sigma$ is based on the net freshwater transport by the MOC into the Atlantic basin. It changes sign as soon as the steady Atlantic MOC enters the multiple equilibrium regime because of an increased freshwater input in the northern North Atlantic. This paper addresses the issue of why the sign of $\Sigma$ is such a good indicator for the multiple equilibrium regime. Changes in the Atlantic freshwater budget over a complete bifurcation diagram and in finite amplitude perturbation experiments are analyzed in a global ocean circulation model. The authors show that the net anomalous freshwater transport into or out of the Atlantic, resulting from the interactions of the velocity perturbations and salinity background field, is coupled to the background (steady state) state freshwater budget and hence to $\Sigma$. The sign of $\Sigma$ precisely shows whether this net anomalous freshwater transport is stabilizing or destabilizing the MOC. Therefore, it can indicate whether the MOC is in a single or multiple equilibrium regime.
\end{abstract}

\section{Introduction}

Over the last few decades, serious concerns have been raised about possible anthropogenic changes in the ocean circulation and the resulting consequences for climate (Rahmstorf 2003). When the ocean velocity field is integrated in an east-west direction across an ocean basin, the resulting flow is referred to as the meridional overturning circulation (MOC). In the Atlantic Ocean, there is a net northward flow of surface and bottom waters, which is compensated for by a southward motion at intermediate depths. The Atlantic MOC transports a substantial amount (about $1.5 \mathrm{PW}$ at $25^{\circ} \mathrm{N}$ ) of heat northward. A reduction (collapse) of the MOC may therefore lead to serious climate change. Regions around the North Atlantic would experience significant cooling, and other parts of the world would also be affected

Corresponding author address: Henk A. Dijkstra, Institute for Marine and Atmospheric Research, Dept. of Physics and Astronomy, Utrecht University, Princetonplein 5, 3584 Utrecht, Netherlands. E-mail: h.a.dijkstra@uu.nl
(Vellinga et al. 2002). Paleoclimatic data suggest that such changes in the MOC may have occurred in the past (Clark et al. 2002; Rahmstorf 2002).

Although wind-generated motions influence the shallow part of the MOC, the deep overturning is dominantly driven by interior turbulent mixing of heat and salt, and it is therefore referred to as the thermohaline circulation (THC). The spatial structure of the THC is predominantly determined by the ocean's density field (Wunsch 2002; Kuhlbrodt et al. 2007). Because the ocean flow itself influences the density field by advection, the THC is by implication a nonlinear phenomenon. Furthermore, the distribution and intensity of surface fluxes of heat and freshwater act to modulate the flow pattern and its temporal behavior.

The nonlinear nature of the THC may result in the existence of multiple equilibria (ME) for a given set of boundary conditions. Under present-day forcing conditions, two stable equilibria appear possible in the models: one usually referred to as the "on" state (or conveyor state) and the other as the "off" state (or collapsed state). Traditionally, large and abrupt changes in the Atlantic 
MOC have been related to the existence of multiple equilibria. These are a robust feature in models ranging from simple box ocean-only models (Stommel 1961) to the so-called earth system models of intermediate complexity (EMIC; Rahmstorf et al. 2005) and have also been found in early coupled climate models (Manabe and Stouffer 1988). It is, however, not clear whether this is a common characteristic of state-of-the-art coupled (oceanatmosphere) general circulation models (CGCMs) simulating present-day climate. In analyzing nine different CGCM simulations of the response to the Intergovernmental Panel on Climate Change (IPCC) Special Report on Emissions Scenarios (SRES) A1B scenario of future $\mathrm{CO}_{2}$ increase, Schmittner et al. (2005), for example, found that none of these models predicted an abrupt change of the MOC.

As it is impossible at the moment to determine whether these CGCMs are in a multiple equilibrium regime, it is important to have (preferably scalar) indicators for the presence of such a regime. Using a simple box model, it was already pointed out by Rahmstorf (1996) that the multiple equilibrium regime may be related to the net freshwater budget over the Atlantic basin. This issue was revisited by De Vries and Weber (2005), who showed (using an EMIC) that the sign of the net freshwater export by the Atlantic MOC, indicated by $M_{\mathrm{ov}}(\theta)$, near the latitude $\theta=35^{\circ} \mathrm{S}$ in the Atlantic may be controlling whether, in addition to the on state, a stable off state exists.

In Dijkstra (2007), it was shown that a measure of the divergence of the freshwater transport $\Sigma$ of the Atlantic MOC over the Atlantic basin is a good indicator of themultiple equilibrium regime. When the freshwater transport at the northern boundary is neglected, $\Sigma$ reduces to the indicator in De Vries and Weber (2005), because

$$
\Sigma\left(\theta_{n}, \theta_{s}\right)=M_{\mathrm{ov}}\left(\theta_{s}\right)-M_{\mathrm{ov}}\left(\theta_{n}\right),
$$

where $\theta_{n}$ and $\theta_{s}$ are the northern and southern latitudes of the Atlantic domain. It appears that $\Sigma$ changes sign (from positive to negative) when the Atlantic MOC on state enters the multiple equilibrium regime as the freshwater input in the northern North Atlantic is increased. There is a slight sensitivity to the choice of the southern boundary, but $\theta_{s}=35^{\circ} \mathrm{S}$ seemingly is a "best" choice because the tip of Africa marks the southern boundary of the Atlantic basin. The sensitivity of $\Sigma$ to the northern-latitude $\theta_{n}$ is relatively small as long as it is north of $60^{\circ} \mathrm{N}$

Although the indicator $\Sigma$ seems able to serve as an indicator for the multiple equilibria regime and descriptive explanations were given in De Vries and Weber (2005) and Dijkstra (2007), there is a need for a better physical basis of this indicator that can explain why $\Sigma$ crosses zero when entering the multiple equilibrium regime and why the southern boundary of $35^{\circ} \mathrm{S}$ is so important. The main purpose of this paper is to provide strong support that $\Sigma$ is a correct indicator for distinguishing different MOC regimes.

The advantage of the approach and methodology followed in Dijkstra (2007) is that full bifurcation diagrams are available and that steady states on the on and off branches satisfy integrated (freshwater) balances with a relative error smaller than $0.1 \%$. In particular, the availability of the unstable steady state-which was shown in Dijkstra et al. (2004) to separate the regions (the so-called attraction basins) of initial conditions going to either on or off states-enables targeted studies on the development of finite amplitude perturbations.

To be self-contained here, we start in section 2 with a very brief summary of the main results in Dijkstra (2007). In section 3, we analyze details of the Atlantic freshwater balance and provide a physical description of the processes that are involved in the changes of this balance along with a full bifurcation diagram. In section 4 , the transient development of specific finite amplitude perturbations is studied with a focus on the processes determining MOC recovery and collapse. The analyses in sections 3 and 4 provide a physical explanation of why the sign of $\Sigma$ is a good indicator for the multiple equilibrium regime. The results are summarized and discussed in section 5. Here, we also address the usefulness of $\Sigma$ in CGCMs and observations and whether it is, in principle, possible to use the indicator to assess the stability regime of the present-day MOC.

\section{The indicator $\Sigma$ in a global ocean model}

Bifurcation diagrams were computed in Dijkstra (2007) using a fully implicit global ocean model. Just to stress that this is no "toy" model, we mention that the governing equations of this ocean model are the hydrostatic, primitive equations in spherical coordinates on a global domain, which includes full continental geometry as well as bottom topography (Dijkstra and Weijer 2005). Horizontal and vertical mixing of momentum and heat and salt is represented by a Laplacian formulation, with prescribed constant "eddy" viscosities $A_{H}$ and $A_{V}$ and vertically dependent "eddy" diffusivities $K_{H}(z)$ and $K_{V}(z)$; see Dijkstra (2007). The ocean flow is forced by the annual-mean wind stress as given in Trenberth et al. (1989). The upper ocean is coupled to a simple energy balance atmospheric model (see the appendix in Dijkstra and Weijer 2005) in which only the heat transport is modeled (no moisture transport). The freshwater flux is prescribed, and the model has no sea ice component. 
Starting from the steady-state solution determined under restoring conditions for the surface salinity field (Levitus 1994), steady states were calculated in Dijkstra (2007) versus a parameter $\gamma_{p}$ under the freshwater flux $F_{S}=P-E$ with

$$
F_{S}=F_{S}^{e}+\gamma_{p} F_{S}^{p}-Q
$$

where $F_{S}^{p}$ is unity only in the domain $(\phi, \theta) \in\left[60^{\circ} \mathrm{W}\right.$, $\left.24^{\circ} \mathrm{W}\right] \times\left[54^{\circ} \mathrm{N}, 66^{\circ} \mathrm{N}\right]$ and zero outside. Furthermore, $F_{S}^{e}$ is freshwater flux diagnosed from the solution at $\gamma_{p}=0$. The quantity $Q$ is determined such that

$$
\int_{S_{o a}} F_{S} r_{0}^{2} \cos \theta d \theta d \phi=0
$$

where $S_{o a}$ is the total ocean surface and $r_{0}$ is the radius of the earth, to ensure a net zero freshwater flux over the total ocean surface.

For the case where the vertical diffusivity $K_{V}$ increases from $1.2 \times 10^{-4} \mathrm{~m}^{2} \mathrm{~s}^{-1}$ at the surface to $5.3 \times$ $10^{-4} \mathrm{~m}^{2} \mathrm{~s}^{-1}$ near the bottom of the flow domain, the bifurcation diagram, where the maximum of the Atlantic MOC $\psi_{A}$ is plotted versus $\gamma_{p}$, is shown in Fig. 1a. For clarity, the stable (drawn) branches are indicated with the on branch and with the off branch. The dashed branch represents unstable steady states. It is clear that the saddle-node bifurcations $L_{-}$and $L_{+}$limit the multiple equilibria regime; we indicate the values of $\gamma_{p}$ at these points with $\gamma_{L-}$ and $\gamma_{L+}$, respectively.

Solutions of the Atlantic MOC along several labeled points of the bifurcation diagram in Fig. 1a are plotted in Figs. 2a-f. For small $\gamma_{p}$, the solution of the Atlantic MOC (the on state) is near to the unperturbed state, with strong northern sinking and no bottom water of southern origin (Fig. 2a). Along the bifurcation diagram the strength of the Atlantic MOC decreases (Fig. 2b), with $\gamma_{p}$ increasing until the saddle-node bifurcation at $\gamma_{L+}$. In the pattern of the Atlantic MOC, the return flow shallows (Fig. 2c) and the deep flow from the south strengthens. Once on the unstable branch of steady states from $L_{+}$to $L_{-}$, this southern sinking component increases, leading eventually to the stable off state (Figs. $2 \mathrm{~d}-\mathrm{f}$ ) for values of $\gamma_{p}>\gamma_{L-}$ on the lower (drawn) branch in Fig. 1. For values of $\gamma_{p}$ between $\gamma_{L-}$ and $\gamma_{L+}$, the MOC is in the ME regime. For $\gamma_{p}<\gamma_{L-}$ and $\gamma_{p}>$ $\gamma_{L+}$, only one stable steady state exists and the MOC is in the single equilibrium (SE) regime.

The indicator $\Sigma$ in Dijkstra (2007) is based on the freshwater budget that arises when the stationary salinity equation is integrated over a volume $V$ of the
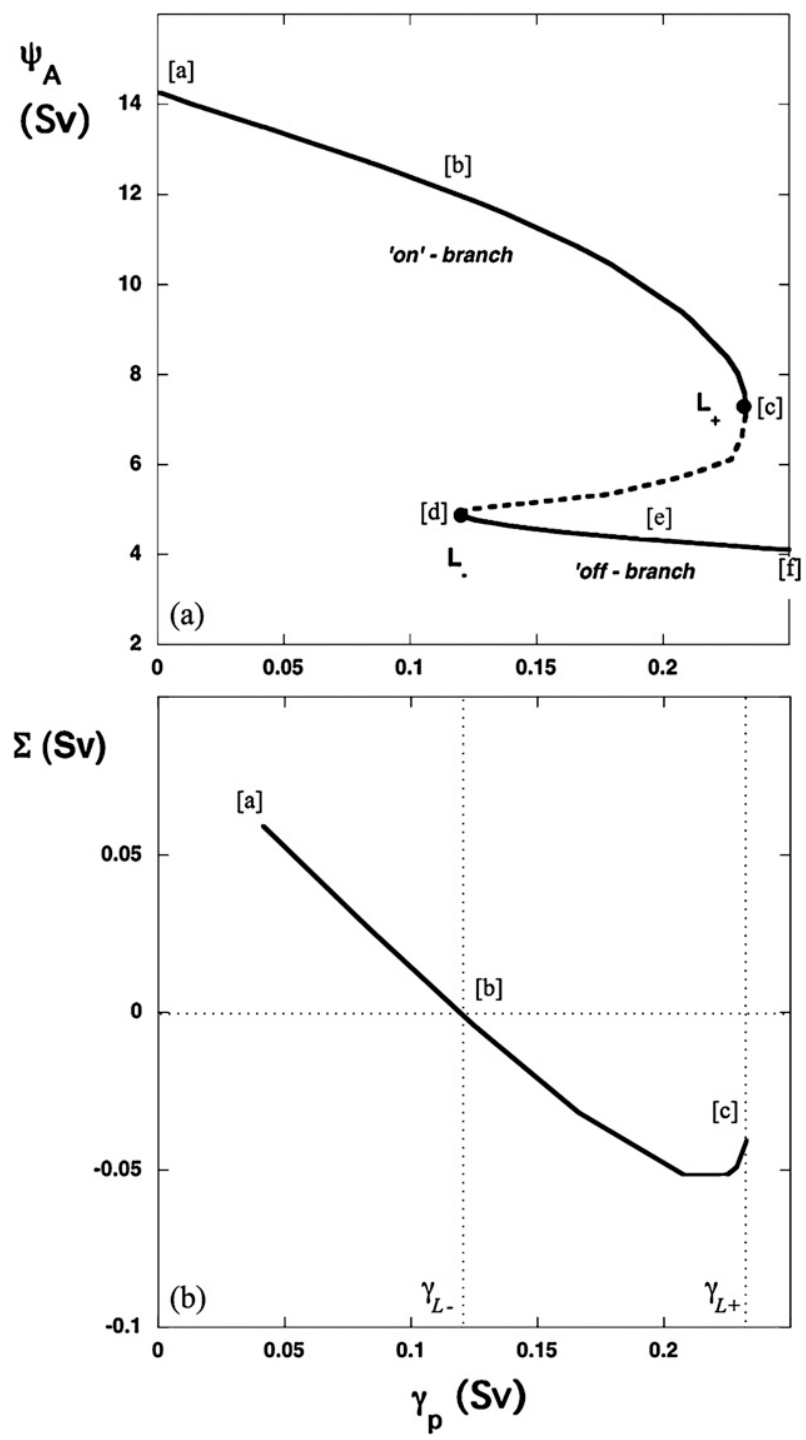

FIG. 1. (a) Bifurcation diagram where the strength of the Atlantic MOC $\left(\psi_{A}\right)$ is plotted vs the strength of the anomalous freshwater forcing $\left(\gamma_{p}\right)$. (a)-(f) The labeling along the branches refers to steady-state solutions of which the meridional overturning is plotted in Fig. 2. (b) Indicator function $\Sigma\left(\theta_{s}, \theta_{n}\right)$ for $\theta_{n}=60^{\circ} \mathrm{N}$ and $\theta_{s}=35^{\circ} \mathrm{S}$ along the on branch of the bifurcation diagram in (a). The vertical dotted lines indicate the positions of $L_{-}$and $L_{+}$.

Atlantic Ocean bounded by the latitudes $\theta_{s}$ and $\theta_{n}$. This integrated salinity budget can be written as

$$
\int_{S_{A l l}} F_{S} r_{0}^{2} \cos \theta d \phi d \theta=-\frac{1}{S_{0}}\left(\Phi\left(\theta_{n}\right)-\Phi\left(\theta_{s}\right)\right)
$$

In (4), the left-hand side is the freshwater volume [in $\mathrm{Sv}\left(1 \mathrm{~Sv} \equiv 10^{6} \mathrm{~m}^{3} \mathrm{~s}^{-1}\right)$ ] going through the oceanatmosphere surface $S_{A t l}$ and the right-hand side is the 

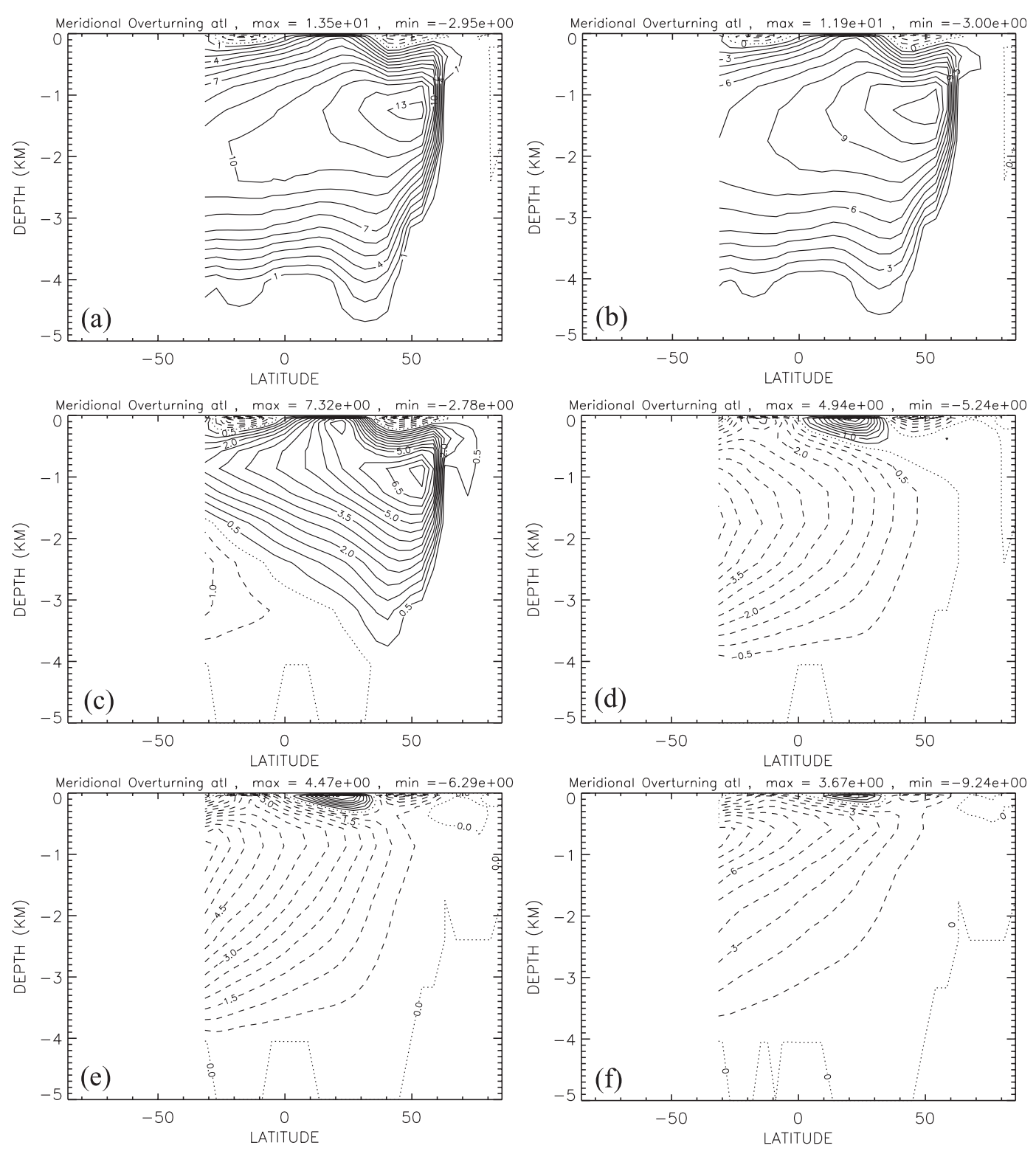

FIG. 2. Contour plots of the Atlantic MOC for several values of $\gamma_{p}$ along the curve in Fig. 1: (a) $\gamma_{p}=0.042, \psi_{A}=$ 13.50 ; (b) $\gamma_{p}=0.125, \psi_{A}=11.87$; (c) $\gamma_{p}=0.234, \psi_{A}=7.32$; (d) $\gamma_{p}=0.121, \psi_{A}=4.90$; (e) $\gamma_{p}=0.170, \psi_{A}=4.44$; and (f) $\gamma_{p}=0.393, \psi_{A}=3.63$ (contour values in Sv).

net freshwater transport (in Sv) through the lateral boundaries at $\theta_{n}$ and $\theta_{s}$. The salt flux $\Phi$ is defined as

$$
\Phi(\theta)=\int_{S_{\theta}}\left(v S-\frac{K_{H}}{r_{0}} \frac{\partial S}{\partial \theta}\right) r_{0} \cos \theta d \phi d z
$$

where $S_{\theta}$ is the (zonal vertical) ocean section at latitude $\theta$.

The indicator $\Sigma\left(\theta_{s}, \theta_{n}\right)$ was already given in (1), where following De Vries and Weber (2005), $M_{\text {ov }}$ (the overturning component) and $M_{\mathrm{az}}$ (the azonal component) are defined as

$$
\begin{aligned}
& M_{\mathrm{ov}}(\theta)=-\frac{\eta}{S_{0}} \int\langle v\rangle\left(\langle S\rangle-S_{0}\right) d z ; \\
& M_{\mathrm{az}}(\theta)=-\frac{\eta}{S_{0}} \int\left\langle v^{\prime} S^{\prime}\right\rangle d z .
\end{aligned}
$$

Here, $\eta$ and $\langle F\rangle$ (for a function $F$ ) are given through

$$
\eta=\int r_{0} \cos \theta d \phi ; \quad\langle F\rangle=\frac{1}{\eta} \int F r_{0} \cos \theta d \phi,
$$

with $v^{\prime}=v-\langle v\rangle$ and $S^{\prime}=S-\langle S\rangle$. The indicator $\Sigma\left(\theta_{s}, \theta_{n}\right)$ is plotted (along the on branch of Fig. 1a) in Fig. 1b for 
$\theta_{n}=60^{\circ} \mathrm{N}$ (in the sinking region) and $\theta_{s}=35^{\circ} \mathrm{S}$ (at the southern tip of Africa). Here, $\Sigma$ changes sign (from positive to negative) just as the multiple equilibrium regime is approached (near the saddle-node bifurcation $\left.L_{-}\right)$. In the SE regime, the Atlantic MOC exports salt $\left(\Sigma\left(\theta_{s}, \theta_{n}\right)>0\right)$, whereas in the ME regime, it is exporting freshwater $\left(\Sigma\left(\theta_{s}, \theta_{n}\right)<0\right)$.

It is remarkable that the indicator $\Sigma$, which is evaluated on the on branch, is able to detect the presence of the saddle-node bifurcation $L_{-}$, which is located on the off branch. Certainly, the on states for $\gamma_{p}>\gamma_{L-}$ are linearly stable (i.e., very small perturbations on these states will decay), but they are susceptible to finite amplitude instabilities. As was shown in Dijkstra et al. (2004), the attraction domains of the on state and off state seem to be bounded by the unstable state, and therefore it requires a finite amplitude perturbation that crosses the unstable branch to make a transition from the on to the off state. But how would $\Sigma$ provide any information on the presence of the multiple equilibrium regime and hence the behavior of finite amplitude perturbations? In the next sections, we will investigate this systematically by analyzing details in the freshwater balances along both the on and off branches (section 3) and by considering the transient development of finite amplitude disturbances on the on state (section 4).

\section{Freshwater balance of the equilibria}

When we represent the diffusive fluxes as

$$
M_{d}(\theta)=\int_{S_{\theta}} \frac{K_{H}}{r_{0}} \frac{\partial S}{\partial \theta} r_{0} \cos \theta d \phi d z
$$

and use the notation

$$
M_{E-P}=\int_{S_{A t l}} S_{0}(E-P) r_{0}^{2} \cos \theta d \phi d \theta
$$

the total freshwater balance (4) can be written as

$$
\begin{gathered}
M_{\mathrm{az}}\left(\theta_{n}\right)+M_{\mathrm{ov}}\left(\theta_{n}\right)+M_{d}\left(\theta_{n}\right)-M_{\mathrm{az}}\left(\theta_{s}\right) \\
\quad+M_{\mathrm{ov}}\left(\theta_{s}\right)-M_{d}\left(\theta_{s}\right)+M_{E-P}=0
\end{gathered}
$$

The terms of this equation are shown in Fig. 3a (southern boundary) and Fig. 3b (northern boundary). As was shown in Dijkstra (2007), the freshwater balance (10) is satisfied accurately in this model. We see that the terms at the northern boundary (Fig. 3b) are smaller than the corresponding terms at the southern boundary (Fig. 3a), also the diffusive terms are not small with respect to the other terms and that many parts of the curves are characterized by near-straight lines. In sections $3 a$ and
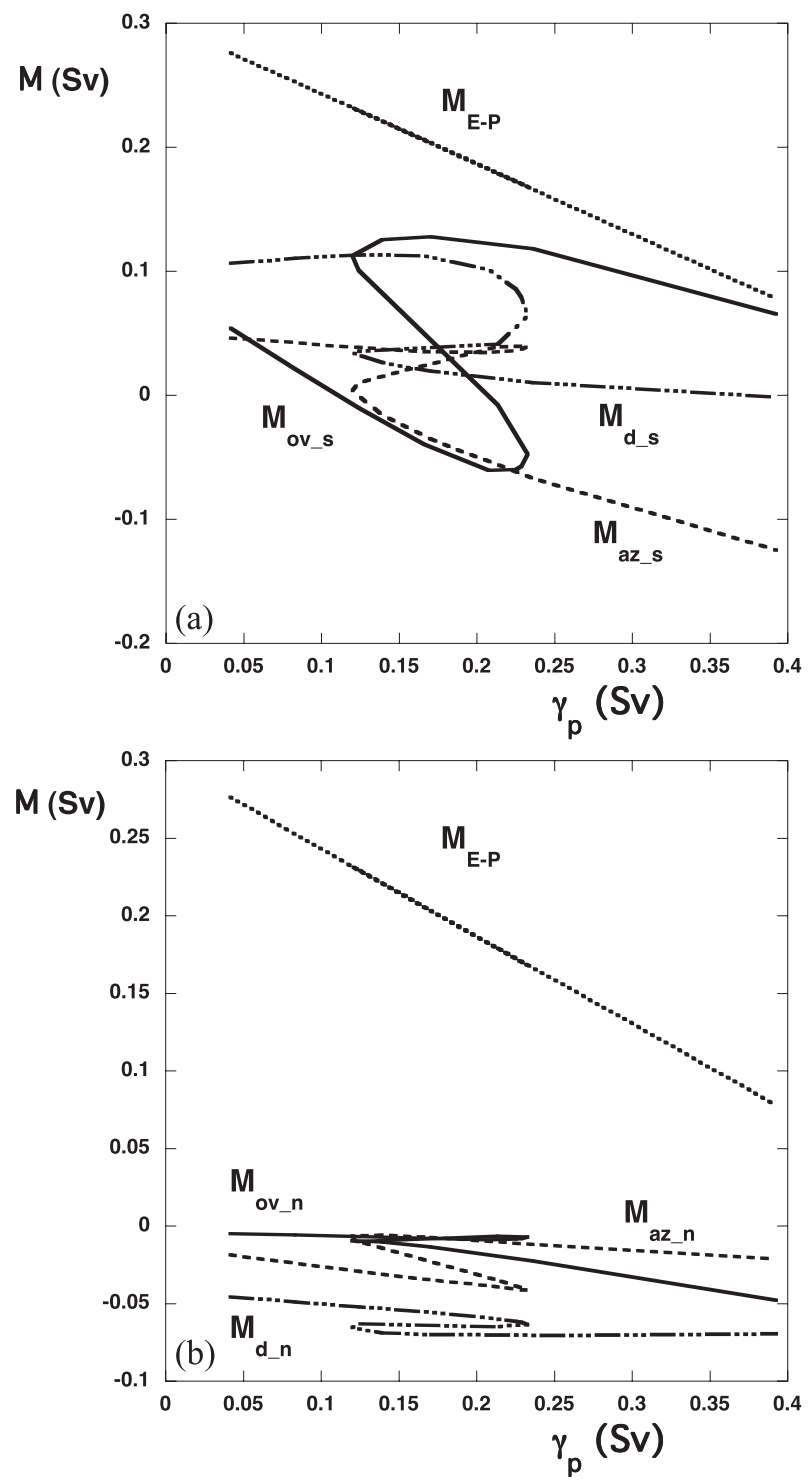

FIG. 3. All terms in the freshwater balance (10) along the bifurcation diagram in Fig. 1: (a) southern boundary $\theta_{s}=35^{\circ} \mathrm{S}$ and (b) northern boundary $\theta_{n}=60^{\circ} \mathrm{N}$ of the Atlantic.

3b, we study the latitudinal dependence of $M_{\mathrm{ov}}$ and $M_{\mathrm{az}}$ on the off and on branches and the change of $M_{\mathrm{ov}}\left(\theta_{s}\right)$ along the on branch.

\section{a. Behavior of $\mathrm{M}_{\mathrm{ov}}$ and $\mathrm{M}_{\mathrm{az}}$ on the off branch}

The function $M_{\mathrm{az}}(\theta)$ is plotted in Fig. 4a. The azonal transport south of $\theta=10^{\circ} \mathrm{S}$ is driven by the subtropical gyre in the South Atlantic, with southward (northward) flow in the western (eastern) part of the basin. The salinity field $S^{\prime}$ (not shown) at the off solution in Fig. 2f for $\theta_{s}=35^{\circ} \mathrm{S}$ is negative in the western part of the basin and positive in the eastern part, the value of $M_{\mathrm{az}}$ is negative and the gyres transport freshwater out of the basin. With 

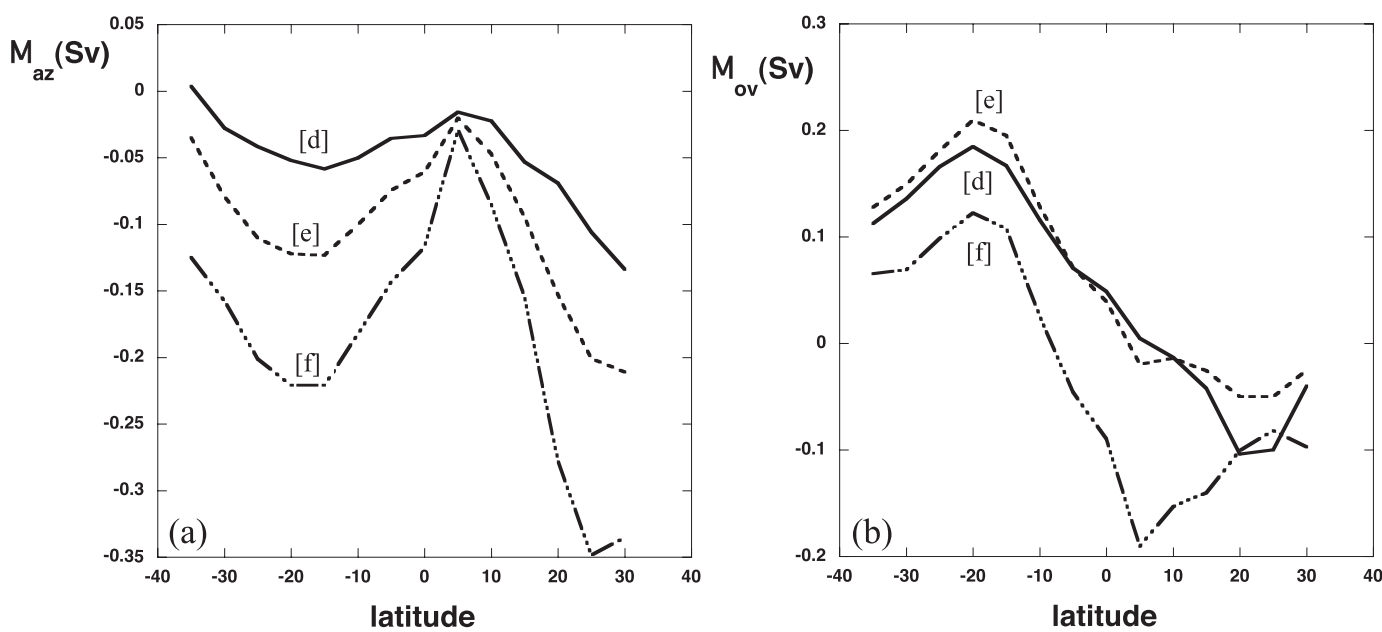

FIG. 4. (a) $M_{\mathrm{az}}(\theta)$ as a function of latitude $\theta$ for the solutions Figs. $2 \mathrm{~d}-\mathrm{f}$ on the off branch. (b) $M_{\mathrm{ov}}(\theta)$ as a function of latitude $\theta$ for the solutions Figs. $2 \mathrm{~d}-\mathrm{f}$ on the off branch.

decreasing $\gamma_{p}$, the salinity field $S^{\prime}$ changes to become more positive at the western side of the basin and more negative at the eastern part. Hence, the value of $M_{\mathrm{az}}$ becomes less negative. The changes in $S^{\prime}$ go down to about $1000 \mathrm{~m}$ while in deeper levels $S^{\prime}$ is hardly affected by the addition of salt in the northern North Atlantic. It is interesting that $M_{\mathrm{az}}$ becomes zero near $\theta=35^{\circ} \mathrm{S}$ at the saddle-node bifurcation $L_{-}$(very close to the solution in Fig. 2d). This indicates that the saddle-node bifurcation, which is a signal of "nonexistence" of a steady solution, is connected to the gyre-driven freshwater transport over the southern boundary.

The function $M_{\mathrm{ov}}(\theta)$ is plotted in Fig. $4 \mathrm{~b}$ and shows that the off state in the Atlantic MOC is exporting salt south of $10^{\circ} \mathrm{S}$. For the solution in Fig. $2 \mathrm{f},\langle v\rangle$ is strongly negative in the upper ocean and the mean salinity $\langle S\rangle-$ $S_{0}>0$, which leads to a positive $M_{\mathrm{ov}}(\theta)$. When $\gamma_{p}$ decreases (effectively adding salt in the northern North Atlantic), the mean salinity at $35^{\circ} \mathrm{S}$ increases over the whole depth and the mean surface velocity decreases slightly, leading to a slight increase in $M_{\mathrm{ov}}(\theta)$ at $\theta=35^{\circ} \mathrm{S}$ (Fig. 4b).

\section{b. Behavior of $\mathrm{M}_{\mathrm{ov}}$ and $\mathrm{M}_{\mathrm{az}}$ on the on branch}

The latitudinal dependence of $M_{\mathrm{az}}$ is plotted in Fig. 5a for the solutions in Figs. 2a-c. South of $\theta=20^{\circ} \mathrm{S}$, the gyre-driven freshwater transport is positive-that is, the gyres transport salt into the basin. Again the velocity distributions $v^{\prime}$ are fairly similar to those along the off branch (because the winds are the same); however, now the salinity distributions $S^{\prime}$ are quite different, with positive values in the western part of the basin and negative ones at the eastern part of the basin. With increasing $\gamma_{p}$ (adding freshwater in the northern North
Atlantic), the fields $S^{\prime}$ hardly change and hence $M_{\mathrm{az}}$ south of $20^{\circ} \mathrm{S}$ remains constant with $\gamma_{p}$. The reason is that the freshwater anomaly is transported southward at depth by the on state and therefore this does not affect the azonal transport.

In Fig. $5 \mathrm{~b}$ the function $M_{\mathrm{ov}}(\theta)$ is positive at the solution in Fig. 2a, which is south of the equator as the ocean velocity at depth $\langle v\rangle$ is negative where the salinity $\langle S\rangle$ is largest. Hence, the meridional overturning transports salt out of the basin. When $\gamma_{p}$ is increased, one sees the decrease in mean salinity at depth, whereas the salinity at the surface increases. This implies that $M_{\mathrm{ov}}$ must decrease with increasing $\gamma_{p}$ as is seen in Fig. 2a.

Actually, it appears that there is linear behavior of $M_{\mathrm{ov}}$ with $\gamma_{p}$ on the on branch, resulting from the specific choice of the forcing. When we differentiate the forcing (2) with respect to $\gamma_{p}$, we find a positive constant $(\alpha)$, and hence

$$
M_{E-P}=M_{E-P}^{0}-\alpha \gamma_{p}
$$

Here, the superscript in $M_{E-P}^{0}$ indicates the solution for $\gamma_{p}=0$, the reference solution. The linear relation in (11) is clearly seen in Fig. 3.

Using the notation $\Sigma_{\mathrm{az}}=M_{\mathrm{az}}\left(\theta_{s}\right)-M_{\mathrm{az}}\left(\theta_{n}\right)$ and $\Sigma_{d}=$ $M_{d}\left(\theta_{s}\right)-M_{d}\left(\theta_{n}\right)$, we can write the Eq. (10) as

$$
\Sigma+\Sigma_{\mathrm{az}}+\Sigma_{d}=M_{E-P}
$$

We argued above that $\Sigma_{\text {az }}$ does not change much along the on branch and it appears from Fig. 3 that the same holds for $\Sigma_{d}$. Using this result, (11) and the fact that $M_{E-P}^{0}=\Sigma^{0}+\Sigma_{\mathrm{az}}^{0}+\Sigma_{d}^{0}$ for the reference solution, we find that 

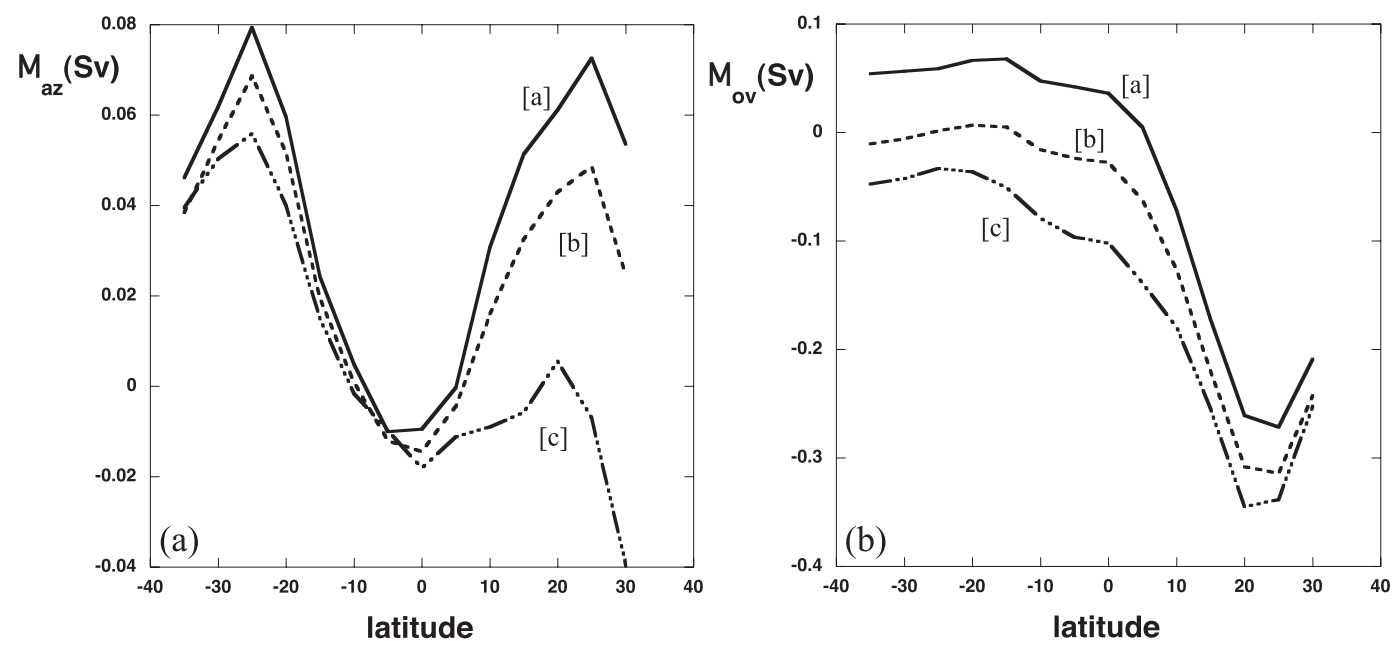

FIG. 5. (a) $M_{\mathrm{az}}(\theta)$ as a function of latitude $\theta$ for the solutions Figs. 2a-c on the on branch. (b) $M_{\mathrm{ov}}(\theta)$ as a function of latitude $\theta$ for the solutions Figs. $2 \mathrm{a}-\mathrm{c}$ on the on branch.

$$
\Sigma \approx-\alpha \gamma_{p}+\Sigma^{0}
$$

This indicates that $\Sigma$ has a zero on the on branch with increasing $\gamma_{p}$ (as $\left.\alpha>0\right)$ when $\Sigma^{0} / \alpha<\gamma_{L+}$. Physically, this simply means that the input of freshwater in the northern North Atlantic eventually leads to the export of freshwater by the Atlantic meridional overturning circulation.

Although from (13) it is clear that $\Sigma$ decreases along the on branch, it is not at all guaranteed that it is changing sign near $\gamma_{L_{-}}$. Therefore, why are the sign changes of the quantities $\Sigma$ and the zonal salinity difference on the on branch associated with the existence (nonexistence) boundary of the off branch? This is a highly nonlinear problem because the issue is whether a finite amplitude perturbation to the on state is able to recover. In the unique regime, one can put any perturbation on the MOC but it will always recover. In the multiple equilibrium regime, there always exists a finite amplitude perturbation that is able (i.e., which is large enough) to induce a transition to the (stable) off state for the same value of $\gamma_{p}$. Hence, an analysis of the development of finite amplitude perturbations in both regimes is required and this is the focus of the next section.

\section{Development of finite amplitude perturbations}

In this section, we investigate why the on state of the MOC always recovers in the SE regime but that in the ME regime the off state can be reached. Thereto, we apply specific finite amplitude freshwater perturbations by considering the so-called thermohaline pulse response problem (similar to the approach in Dijkstra et al. 2004), where the freshwater perturbation is switched on instantly and after a certain time $t_{m}$ is suddenly reduced to zero. If we represent the time dependence as a block function $B\left(t ; t_{m}\right)$, then the total freshwater flux can be written as

$$
F_{S}=F_{S}^{e}+\left(\bar{\gamma}_{p}+\Delta \gamma_{p} B\left(t ; t_{m}\right)\right) F_{S}^{p}-Q(t) \quad \text { and }
$$

$B\left(t ; t_{m}\right)=H(t)-H\left(t-t_{m}\right)$,

where $H$ is the Heaviside function and $Q(t)$ is determined from the condition that the surface integrated freshwater flux is zero. The value of $\bar{\gamma}_{p}$ refers to the value of $\gamma_{p}$ at a steady state and we use $\Delta \gamma_{p}$ as a perturbation of it. To facilitate the interpretation of the results, we choose to make the pattern of the freshwater perturbation associated with $\Delta \gamma_{p}$ the same as that was computed for the bifurcation diagram (in $\gamma_{p}$ ).

\section{a. Transient solutions}

Now, let us assume we start from a steady-state solution determined for a certain $\bar{\gamma}_{p}$ on the on branch. When $\Delta \gamma_{p}=0$, we will remain at that steady state. However, when $\Delta \gamma_{p}$ is so large that

$$
\gamma_{p}=\bar{\gamma}_{p}+\Delta \gamma_{p}>\gamma_{L+},
$$

the solution will be attracted to the off state for $\bar{\gamma}_{p}+\Delta \gamma_{p}$ because this is the only steady state for this value of $\gamma_{p}$. After a time $t_{m}$, the trajectory will reach a certain state and when the anomalous forcing is then suddenly released, the trajectory will be attracted to one of the stable steady states that are present for $\gamma_{p}=\bar{\gamma}_{p}$. In the SE regime there is only one steady state at $\bar{\gamma}_{p}$ and all trajectories (for all values of $t_{m}$ ) will be attracted to the 

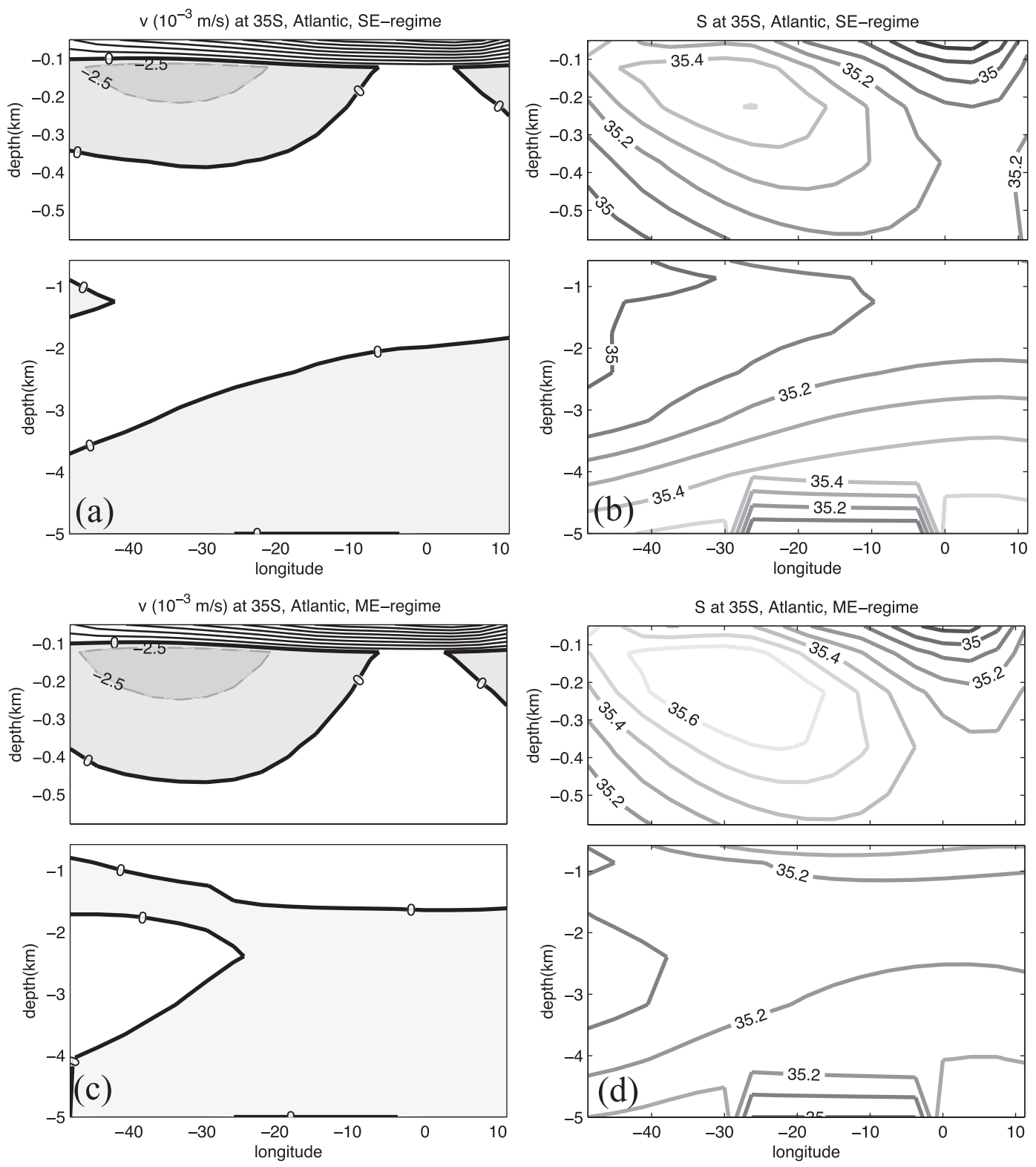

FIG. 6. Contour plots of (a),(c) the meridional velocity $\bar{v}$ and (b),(d) the salinity $\bar{S}$ field along the Atlantic $35^{\circ} \mathrm{S}$ section for both (a),(b) the steady state in the SE regime $\left(\bar{\gamma}_{p}^{S}=0.083 \mathrm{~Sv}\right)$ and (c),(d) the steady state in the ME regime $\left(\bar{\gamma}_{p}^{M}=0.166 \mathrm{~Sv}\right)$. Negative values are shaded.

on state, the MOC always recovers. In the ME regime, however, the MOC will recover for a small $t_{m}$, whereas the off state will be reached for a large $t_{m}$. In Dijkstra et al. (2004), it was shown that the critical time, that is $t_{m}^{*}$, is determined by the time when the unstable steady state is crossed.

We take a value $\bar{\gamma}_{p}^{S}=0.083 \mathrm{~Sv}$ in the SE regime and a value $\bar{\gamma}_{p}^{M}=0.166 \mathrm{~Sv}$ in the ME regime. Plots of the meridional velocity field and salinity field at $35^{\circ} \mathrm{S}$ are presented in Fig. 6 for both steady states. The meridional velocity fields look very similar for both cases, al- though the MOC is slightly shallower in Fig. $6 c$ than in Fig. 6a. The salinity fields differ markedly, with a much larger salinity contrast between surface and deep ocean in the ME regime. This reflects the change in freshwater transport by the MOC over this section, which is positive (freshwater transport into the Atlantic basin) in the SE regime and negative (freshwater transport out of the basin) in the ME regime.

To analyze the development for a relatively small $\Delta \gamma_{p}$, we show results for both cases when the states in Fig. 6 undergo a change of $\Delta \gamma_{p}^{S}=\Delta \gamma_{p}^{M}=0.02 \mathrm{~Sv}$ for $t_{m}=200 \mathrm{yr}$. 

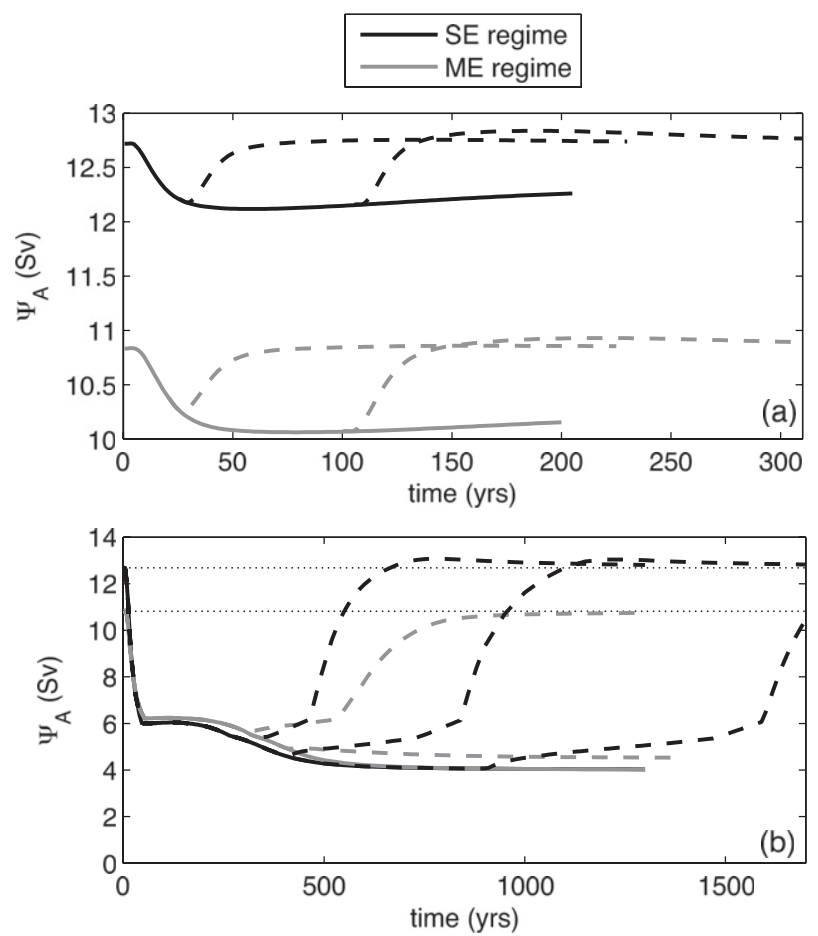

FIG. 7. Strength $\left(\psi_{A}\right)$ in Sv of the Atlantic MOC along trajectories (drawn) for a permanent freshwater flux change for both the $\mathrm{SE}$ (black) regime $\left(\bar{\gamma}_{p}^{S}=0.083 \mathrm{~Sv}\right.$ ) and the $\mathrm{ME}$ (gray) regime $\left(\bar{\gamma}_{p}^{M}=0.166 \mathrm{~Sv}\right)$. The dashed lines are trajectories for which the freshwater flux change is reduced to zero at certain times $t_{m}$; the latter time can be deduced from the starting point of each dashed curve. (a) A very small change, $\Delta \gamma_{p}^{S}=\Delta \gamma_{p}^{M}=0.02$ Sv. (b) A larger change, $\Delta \gamma_{p}^{S}=0.197 \mathrm{~Sv}$ and $\Delta \gamma_{p}^{M}=0.114 \mathrm{~Sv}$.

The maximum of the Atlantic $\operatorname{MOC}\left(\psi_{A}\right)$ is plotted versus time in Fig. 7a and the drawn curves (black, SE regime; gray, ME regime) show the long time development under this change in freshwater flux. After about 200 years, the MOC strength has decreased less than $1 \mathrm{~Sv}$ for both cases. When the anomalous forcing is released at $t_{m}=20$ and $t_{m}=100 \mathrm{yr}$ (dashed curves), the original equilibria are obtained showing that the steady states are indeed linearly stable.

Subsequently, we take $\Delta \gamma_{p}^{S}=0.197 \mathrm{~Sv}$ and $\Delta \gamma_{p}^{M}=$ $0.114 \mathrm{~Sv}$, respectively, such that for $t_{m} \rightarrow \infty$ the same off state at $\gamma_{p}=0.280 \mathrm{~Sv}$ is reached (cf. Fig. 1). This is confirmed by the drawn curves in Fig. $7 \mathrm{~b}$. The dashed curves in Fig. 7b again show the development of the MOC strength, when the anomalous forcing is turned to zero (i.e., $\gamma_{p}=\bar{\gamma}_{p}$ ) after a time $t_{m}$. It is indeed seen that for every $t_{m}$ the trajectories in the SE regime (black curves) eventually end up on the on state. However, for the ME regime (gray curves) we see that for $t_{m}<t_{m}^{*}$ the Atlantic MOC recovers but that for $t_{m}>t_{m}^{*}$ the Atlantic MOC approaches the off state; here is the critical time $t_{m}^{*} \approx 400 \mathrm{yr}$.
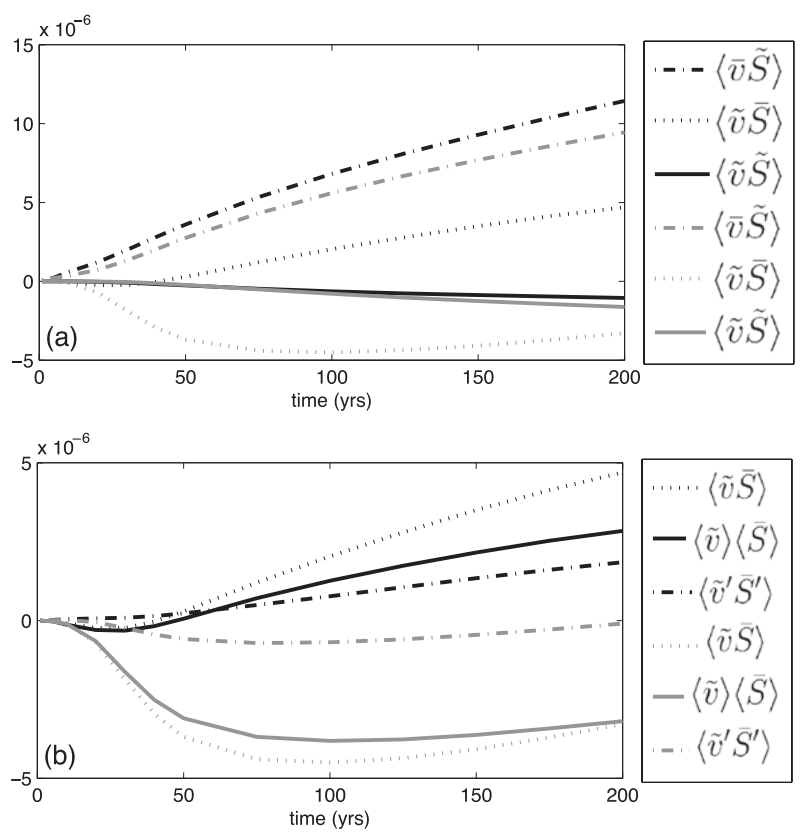

FIG. 8. (a) Time development of the three different terms in the rhs of (17) along the drawn trajectories in Fig. 7a. The color coding corresponds to that in Fig. 7a (black; SE regime and gray; ME regime) and a similar line style indicates a similar term. (b) Integral terms in (18). The dotted curve is again the $\tilde{v} \bar{S}$ integral term similar to the one in (a). The drawn (dashed-dotted) curve is the first (second) term in the rhs of (18).

\section{b. Analysis}

We now turn to a physical explanation of the results in Fig. 7. Our task is to explain why 1) the MOC recovers in the ME regime for $t_{m}<t_{m}^{*}$, and collapses for $t_{m}>t_{m}^{*}$ and 2) the MOC in the SE regime, when subjected to a similar perturbation, always recovers. Our ingredients are that $\Sigma \approx M_{\mathrm{ov}}\left(\theta_{s}\right)<0$ for the steady on state in the ME regime and $\Sigma \approx M_{\mathrm{ov}}\left(\theta_{s}\right)>0$ in the SE regime.

The existence of multiple equilibria in the MOC is caused by the salt-advection feedback, which is present in its simplest form in the Stommel (1961) box model. A freshwater perturbation on the on state of the MOC, for example, causes a weakening of the MOC and hence leads to a smaller meridional advective salt transport, which amplifies the original perturbation. As the advective meridional transport is crucial, this feedback has also been identified in two- and three-dimensional ocean models (Walin 1985; Dijkstra and Molemaker 1997; Kuhlbrodt et al. 2007).

If a perturbation $(\tilde{v}, \tilde{S})$ is assumed on a mean state $(\bar{v}, \bar{S})$, the development of the salinity perturbation is determined [considering only meridional advective transport and hence neglecting all other (zonal/vertical, advection, and diffusive) transports] by the equation 

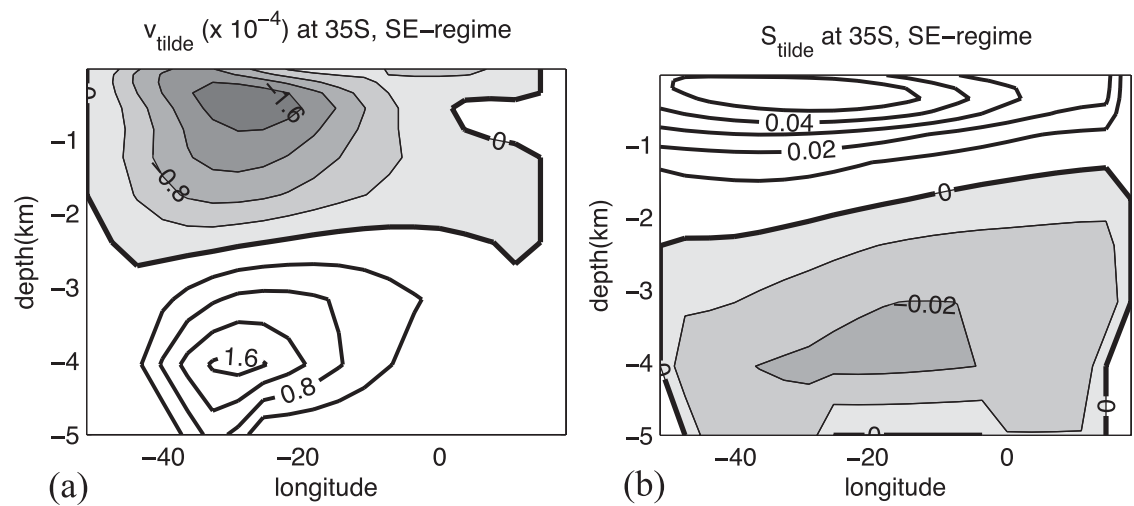

$\mathrm{v}_{\text {tilde }}\left(\times 10^{-4}\right)$ at $35 \mathrm{~S}, \mathrm{ME}-$ regime
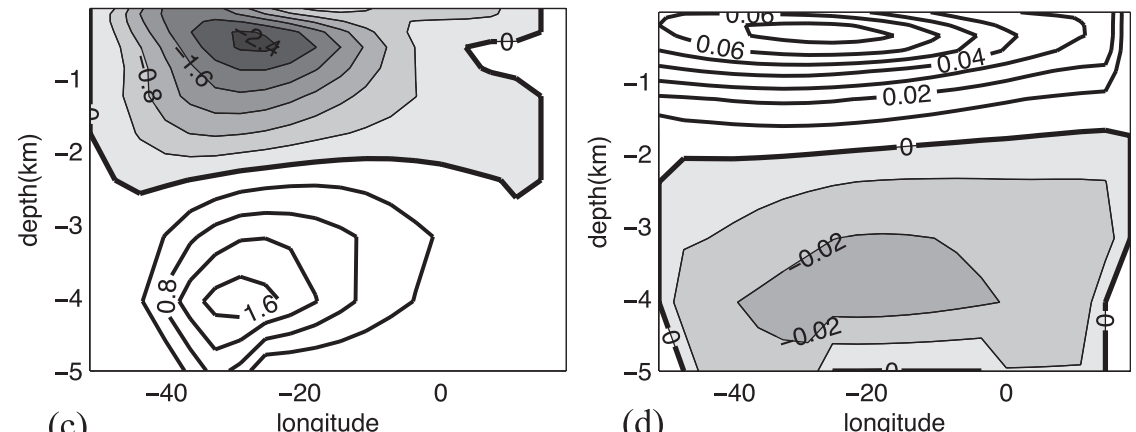

(d)

FIG. 9. Contour plots of (a),(c) the meridional velocity and (b),(d) the salinity perturbations at the year 200 field along the Atlantic $35^{\circ} \mathrm{S}$ section for both the SE regime $\left(\bar{\gamma}_{p}^{S}=0.083 \mathrm{~Sv}\right)$ and the ME regime $\left(\bar{\gamma}_{p}^{M}=0.166 \mathrm{~Sv}\right)$ under the change $\Delta \gamma_{p}^{S}=\Delta \gamma_{p}^{M}=0.02 \mathrm{~Sv}$. Negative values are shaded.

$$
\frac{\partial \tilde{S}}{\partial t} \approx-\frac{1}{r_{0}}\left[\tilde{v} \frac{\partial \bar{S}}{\partial \theta}+\bar{v} \frac{\partial \tilde{S}}{\partial \theta}+\tilde{v} \frac{\partial \tilde{S}}{\partial \theta}\right] .
$$

When we integrate (16) over the Atlantic basin (longitude $\phi$ from coast to coast, $z$ from bottom to surface, and latitude $\theta$ from $\theta_{n}$ to $\theta_{s}$ ), then the development of the Atlantic basin averaged salinity anomaly is governed by the equation

$$
\frac{\partial}{\partial t}\left(\int_{V} \tilde{S} d^{3} x\right) \approx \int_{S_{\theta_{S}}}(\tilde{v} \bar{S}+\tilde{S} \bar{v}+\tilde{v} \tilde{S}) d^{2} x
$$

where the fluxes through the northern boundary are neglected. This relation shows that the growth of the salinity anomaly in the Atlantic basin is related to the anomalous meridional salt transport terms integrated over the southern boundary.

In the following, we will investigate the time development of the different terms in the right-hand side of (17) along the trajectories in Fig. 7a. We will call the contribution of a certain term in the (17) "stabilizing" (destabilizing) when it increases (decreases) the salt content of the Atlantic basin and hence strengthens (weakens) the MOC. The terms are plotted in Fig. 8a for $\Delta \gamma_{p}=0.02 \mathrm{~Sv}$. Note that because this value of $\Delta \gamma_{p}$ is relatively small, both linear interaction terms are of larger magnitude than the nonlinear interaction term $\tilde{v} \tilde{S}$. The $\bar{v} \tilde{S}$ term is positive for both SE and ME regimes and this transport is stabilizing the MOC. The nonlinear interaction term is negative (making the Atlantic fresher) and hence is destabilizing the MOC for both regimes. The central result is that the $\tilde{v} \bar{S}$ term is stabilizing in the SE regime, whereas it is destabilizing for the ME regime.

To understand the sign of the terms in (17), plots of $\tilde{v}$ and $\tilde{S}$ are shown in Fig. 9 for the solutions at year 200 (endpoints of drawn curves in Fig. 7a). For both ME and SE regimes, the $\tilde{S}$ field is positive at the surface and negative at depth, which is a typical response to the slowdown of the MOC. As the $\bar{v}$ term is largest at the surface (cf. Fig. 6a), the anomalous salt transport associated with the term $\bar{v} \tilde{S}$ is into the basin and hence is stabilizing. As $\tilde{v}$ is negative at the surface and positive at depth (Figs. 9a,c), the nonlinear interaction term $\tilde{v} \tilde{S}$ always leads to salt export out of the Atlantic basin and hence this term is destabilizing.

The $\tilde{v} \bar{S}$ field is plotted for the SE and ME regimes (again at year 200) in the Figs. 10a,b, respectively, and 

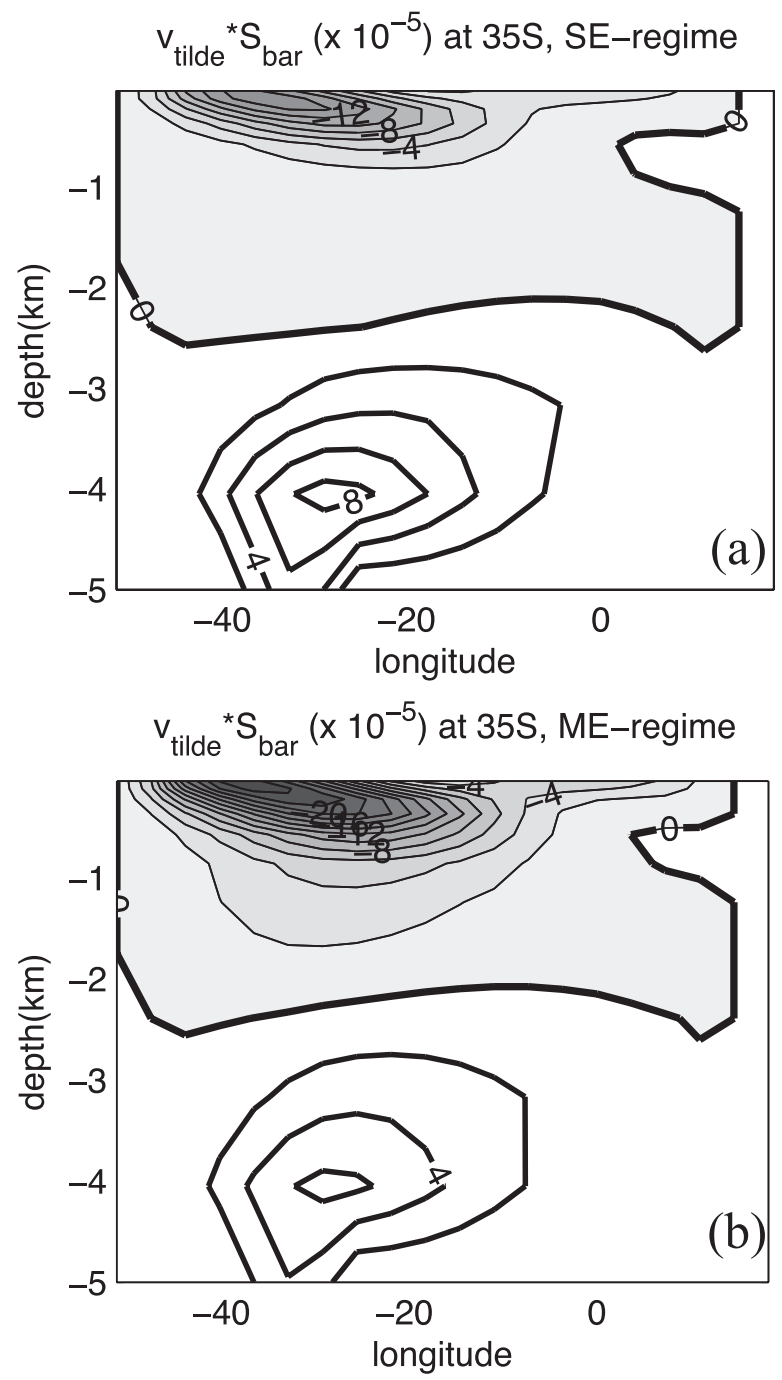

$\mathrm{v}_{\text {tilde }}{ }^{*} \mathrm{~S}_{\text {bar }}\left(\times 10^{-5}\right)$ at 35S, ME y200 - SE y200

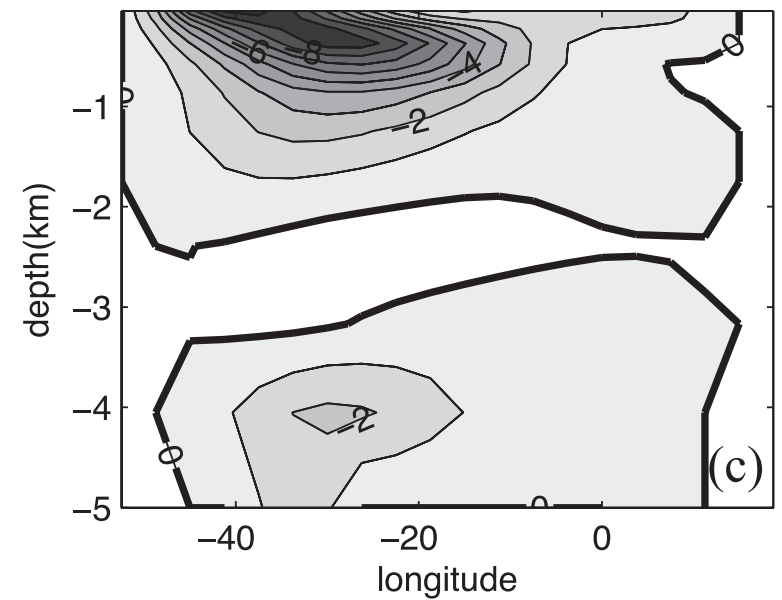

FIG. 10. The field $\tilde{v} \bar{S}$ for (a) the $\mathrm{SE}$ regime, (b) the ME regime, and (c) their difference. The fields are for the solutions at year 200 (drawn curves) in Fig. 7a. Negative values are shaded. their difference is shown in Fig. 10c. The change in the steady-state salinity field with $\gamma_{p}$ turns out to be crucial for the sign of the $\tilde{v} \bar{S}$ term. The salinity $\bar{S}$ is smaller in the deep ocean (below $\sim 3 \mathrm{~km}$ ) for the solution in the ME regime than for the SE regime. Hence, the transport of salt because of the $\tilde{v} \bar{S}$ term is out of the Atlantic basin in the ME regime and into the Atlantic basin for the SE regime; hence, this term is stabilizing in the SE regime and destabilizing in the ME regime.

We can make the link of the section integral over $\tilde{v} \bar{S}$ and $M_{\mathrm{ov}}\left(\theta_{s}\right)$ more explicit by looking at the relation between the profiles of $\langle\bar{v}\rangle$ (Fig. 11a) and $\langle\tilde{v}\rangle$ (plotted for different times in Fig. 11b). By inspection, it appears that for both regimes it is a reasonable assumption that $\langle\tilde{v}\rangle \approx-\epsilon(t)\langle\bar{v}\rangle$. Physically, this makes sense because the MOC decreases as a result of the imposed change in freshwater flux in the northern North Atlantic, and the overall spatial pattern of the MOC remains the same for small $\Delta \gamma_{p}$. In other words, there is a southward velocity perturbation in the upper layers and a northward velocity perturbation at depth.

When we now decompose $\tilde{v}=\langle\tilde{v}\rangle+\tilde{v}^{\prime}, \bar{S}=\langle\bar{S}\rangle+\bar{S}^{\prime}$, then the section integral can be written as

$$
\begin{aligned}
\int_{z} \int_{\phi} \tilde{\boldsymbol{v}} \bar{S} r_{0} \cos \theta d \phi d z= & \eta \int_{z}\langle\tilde{\boldsymbol{v}}\rangle\langle\bar{S}\rangle d z \\
& +\int_{z} \int_{\phi} \tilde{\boldsymbol{v}}^{\prime} \bar{S}^{\prime} r_{0} \cos \theta d \phi d z .
\end{aligned}
$$

Using $\langle\tilde{v}\rangle \approx-\epsilon(t)\langle\bar{v}\rangle$, the first term in the right-hand side is proportional to $M_{\mathrm{ov}}\left(\theta_{s}\right)$. The second integral is dependent on the azonal components of $\tilde{v}$ and $\bar{S}$. For both $\mathrm{SE}$ and ME cases in Fig. 8a, the three integrals in (18) are plotted in Fig. 8b. For the ME regime (gray curves) the integral involving the $\tilde{v}^{\prime} \bar{S}^{\prime}$ term is much smaller than that involving the $\langle\tilde{\boldsymbol{v}}\rangle\langle\bar{S}\rangle$ term. Hence, the sign of the section integral of $\tilde{v} \bar{S}$ is the same as that of $M_{\mathrm{ov}}$ at $35^{\circ} \mathrm{S}$. For the SE case, the integrals in the righthand side of (18) are of the same order of magnitude, so here the relation between the sign of the $\tilde{v} \bar{S}$ integral and $M_{\mathrm{ov}}$ at $35^{\circ} \mathrm{S}$ is less obvious. However, from Fig. $8 \mathrm{~b}$, it is clear that the sign of the $\tilde{v} \bar{S}$ term is most influenced by the changes in the sign of the $\langle\tilde{v}\rangle\langle\bar{S}\rangle$ term.

Furthermore, from Fig. 8a it can be seen that the stabilizing term $\bar{v} \tilde{S}$ decreases and the destabilizing term $\tilde{v} \tilde{S}$ becomes more negative (more destabilizing) with increasing $\gamma_{p}$. This demonstrates that when the on state of the MOC enters the ME regime, the salt transport near the southern boundary induced by changes in the northern North Atlantic freshwater flux tends to be more destabilizing. In the previous analysis, the salt transport at the northern boundary $\theta_{n}$ is assumed to be 

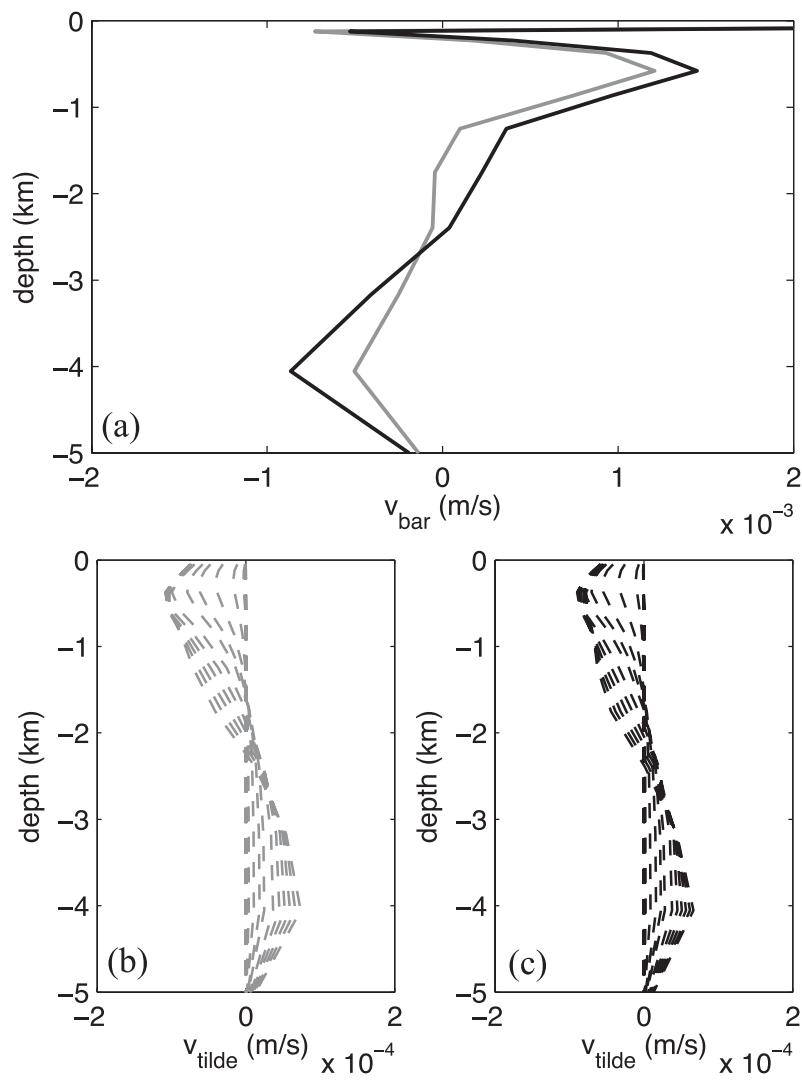

FIG. 11. (a) The field $\langle\bar{v}\rangle$ for the steady state at $\bar{\gamma}_{p}^{S}=0.083 \mathrm{~Sv}$ (black) and $\bar{\gamma}_{p}^{M}=0.166 \mathrm{~Sv}$ (gray). The fields $\langle\tilde{\boldsymbol{v}}\rangle$ at different times (every 25 years up to year 200) for (b) the ME regime and (c) the SE regime. These fields are computed for the solutions along the drawn curves in Fig. 7a.

much smaller than that at the southern boundary $\theta_{s}$ but it can easily be taken into account in (17). Following (18), the integral of the term $\tilde{v} \bar{S}$ over the northern and southern boundary can then be related to $\Sigma$. Hence, assuming a dominance of the meridional advective transport terms, the sign change in the $\tilde{v} \bar{S}$ term, as directly linked to $\Sigma$, is the crucial effect determining whether the MOC is in the SE or ME regime. When the perturbations become very large, as in the results of Fig. $7 \mathrm{~b}$, the pattern of the MOC changes drastically with time; eventually, the magnitudes of all terms in (17) along the drawn trajectories in Fig. $7 \mathrm{~b}$ will determine whether the MOC will collapse or recover when the forcing is released.

\section{c. Further analysis in a box model}

To substantiate the reasoning in the last section, we analyze the same connection between properties of the steady states and the transient development of perturbations in the Rahmstorf (1996) model. In this box model, only the meridional advective processes are taken into account. The model consists of two equatorial boxes connected to two polar boxes that are arranged as depicted in Fig. 12a. It is designed to mimic the pattern of the Atlantic circulation, with deep convection at subpolar latitudes (box 2), while low-latitude upwelling is limited (hence no connection between boxes 3 and 4) but instead takes place outside the domain of the Atlantic (box 1). The volume transport between the boxes has strength $m$ and is proportional to the density difference $\rho_{2}-\rho_{1}$. Assuming a linear equation of state, the flow strength is diagnosed from

$m=k\left(\rho_{2}-\rho_{1}\right)=k\left[\beta\left(S_{2}-S_{1}\right)-\alpha\left(T_{2}-T_{1}\right)\right]$,

where $k, \beta$, and $\alpha$ are constants and $T_{i}$ and $S_{i}$ are the temperature and salinity in box $i$.

Mixed boundary conditions are imposed where temperatures will be simply prescribed and the surface freshwater forcing consists of two independent active fluxes, $F_{1}$ and $F_{2}$. The adjective "active" here means that not only atmospheric vapor fluxes are captured in the $F_{i}$, but also the salt transport by the wind-driven gyres as well as (subgrid scale) diffusion. The fluxes $F_{i}$ are converted to equivalent salt transports by multiplication with $-S_{0}$, a fixed reference salinity, and will be assumed constant. The salt conservation equation for the southern box is then given by

$$
V_{1} \frac{d S_{1}}{d t}=\left\langle\begin{array}{l}
m\left(S_{4}-S_{1}\right) \\
m\left(S_{1}-S_{3}\right)
\end{array}\right\rangle+S_{0} F_{1} \quad \text { for } \quad \begin{aligned}
& m \geq 0 \\
& m \leq 0
\end{aligned}
$$

Here, $V_{1}$ is the fixed volume of box 1 . The equations for the other boxes follow in a similar fashion (Rahmstorf 1996).

For $m \geq 0$, the steady-state salinity in box 2 is equal to that in box $4, \bar{S}_{2}=\bar{S}_{4}$. The combination of Eqs. (19) and (20) then yields the steady-state flow strength as function of the temperature contrast and the freshwater forcing:

$$
\begin{aligned}
& \bar{m}=-\frac{1}{2} k \alpha\left(T_{2}-T_{1}\right) \pm \sqrt{\frac{1}{4}\left[k \alpha\left(T_{2}-T_{1}\right)\right]^{2}-k \beta S_{0} F_{1}} \\
& \text { for } \bar{m} \geq 0 .
\end{aligned}
$$

For $\bar{m} \leq 0$, the solution is similar to (22) but with $-F_{1}$ replaced by $+F_{2}$.

We will only consider the case for which $T_{1}>T_{2}$. For $\bar{m} \geq 0$, solutions exist for $F_{1} \in\left(-\infty, F_{1}^{\text {Crit }}\right)$, where $F_{1}^{\text {Crit }}=k \alpha^{2}\left(T_{2}-T_{1}\right)^{2} / 4 \beta S_{0}$, the critical freshwater flux at the saddle-node bifurcation. We are primarily interested in the transition associated with the sign change 

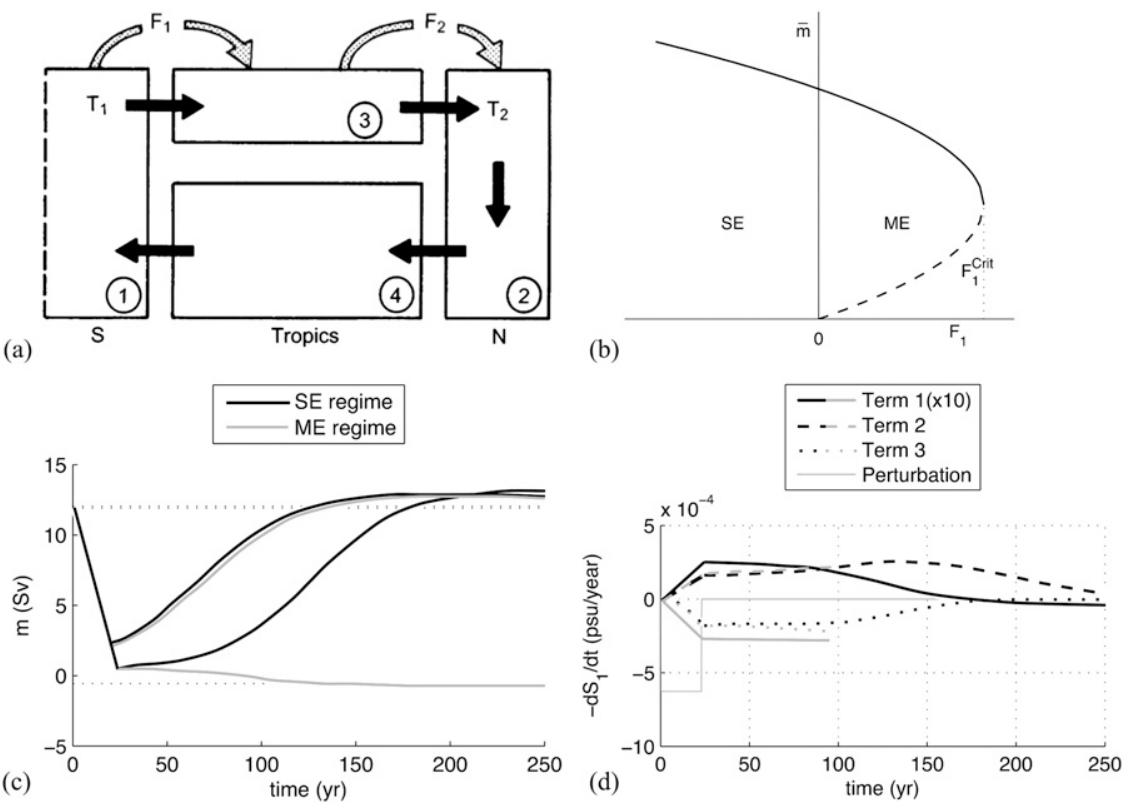

(c)

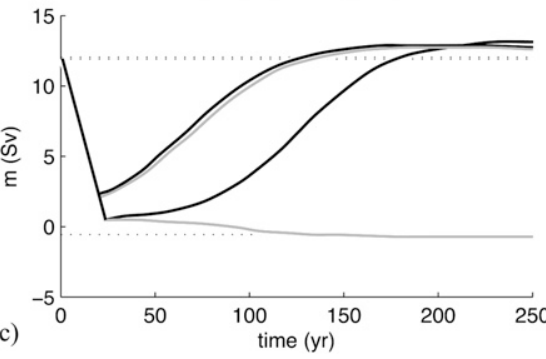

FIG. 12. (a) Simple box model of the Atlantic overturning circulation (Rahmstorf 1996). Deep convection takes place in box 2 . The strength of the cross-equatorial flow is controlled by the density contrast between boxes 2 and 1 . The temperatures $T_{1}$ and $T_{2}$ are prescribed, while salinities are determined by the flow and the active freshwater fluxes $F_{1}$ and $F_{2}$. (b) Bifurcation diagram showing the steady solutions with positive transport vs $F_{1}$. (c) Overturning strength $m$ as function of time for the four perturbation experiments. (d) Negative time rate of change of the salinity in box 1 as function of time, split into the three contributions given in Eq. (22): term 1 corresponds to $\tilde{m}\left(\bar{S}_{4}-\bar{S}_{1}\right)$, term 2 to $\bar{m}\left(\tilde{S}_{4}-\tilde{S}_{1}\right)$, and term 3 to $\tilde{m}\left(\tilde{S}_{4}-\tilde{S}_{1}\right)$.

of $F_{1}$, which Rahmstorf (1996) identified with the transition from the single equilibrium to the multiple equilibrium regime. The bifurcation diagram for this case is plotted in Fig. 12b, showing all steady solutions for $\bar{m} \geq 0$. Note that for $\bar{m} \leq 0$ solutions exist for $F_{2} \geq 0$, independent of $F_{1}$. However, this inverse circulation implies that all upwelling takes place in the northern box, despite the absence of a physical mechanism to limit dominant upward motion to the northern high latitudes. Hence, the applicability of the box model ends when the flow reverses sign. For simplicity we define any situation for which $\bar{m}<0$ as the collapsed state, and we take $F_{2}>0$ to ensure that the trajectories are attracted to this state when $m$ becomes negative.

Suppose we perturb the equilibrium given by Eq. (21) by applying an anomalous freshwater flux of strength $\gamma_{p}$ to box 2 for a time $t_{m}$. Simultaneously, an equal amount of water is extracted from boxes 1 and 3 , in a ratio defined by their respective surface areas $A_{1}$ and $A_{3}$, in order to preserve salinity. This will change the salinity contrast between boxes 1 and 2 such that the flow is weakened, but $\gamma_{p}$ and $t_{m}$ are chosen such that $m$ does not reverse sign during the application of the perturbation. In the first two experiments, $F_{1}$ is taken slightly negative $\left(F_{1}=-10^{-3} \mathrm{~Sv}\right)$, and in the latter two slightly positive
$\left(F_{1}=10^{-3} \mathrm{~Sv}\right)$. For each of the two cases we apply a perturbation $\gamma_{p}=0.25 \mathrm{~Sv}$ for a period of $t_{m}=20 \mathrm{yr}$ and for a period of $t_{m}=23 \mathrm{yr}$. The time scale can be easily increased by changing the parameters as summarized in Table 1 and is not essential here. Figure 12c shows that the system recovers after both perturbations when $F_{1}<0$, which is in the SE regime. On the other hand, when $F_{1}>0$, the system recovers for $t_{m}=20 \mathrm{yr}$ but collapses for $t_{m}=23 \mathrm{yr}$.

The fluxes and salinities may be written as the sum of a mean state $\left(\bar{m}, \bar{S}_{i}\right)$ and a perturbation $\left(\tilde{m}, \tilde{S}_{i}\right)$. Since total salinity is conserved, the evolution of the Atlantic salinity is proportional to $-d \tilde{S}_{1} / d t$, which for positive $m$ is given by

$$
V_{1} \frac{d \tilde{S}_{1}}{d t}=\tilde{m}\left(\bar{S}_{4}-\bar{S}_{1}\right)+\bar{m}\left(\tilde{S}_{4}-\tilde{S}_{1}\right)+\tilde{m}\left(\tilde{S}_{4}-\tilde{S}_{1}\right)
$$

TABLE 1. Values of parameters used in the numerical calculations for the box model.

$k=2.7 \times 10^{4} \quad$ Sv $\quad S_{0}=35.0 \quad$ psu $\quad V_{1}=4.0 \times 10^{16} \mathrm{~m}^{3}$

$\alpha=1.0 \times 10^{-4} \mathrm{~K}^{-1} \quad F_{2}=1.0 \times 10^{-2} \quad \mathrm{~Sv} \quad V_{2}=1.0 \times 10^{16} \mathrm{~m}^{3}$

$\beta=7.6 \times 10^{-4}[] \quad A_{1}=1.0 \times 10^{13} \mathrm{~m}^{2} \quad V_{3}=1.0 \times 10^{17} \mathrm{~m}^{3}$

$T_{1}-T_{2}=4.5 \quad \mathrm{~K} \quad A_{3}=1.0 \times 10^{14} \mathrm{~m}^{2} \quad V_{4}=2.0 \times 10^{17} \mathrm{~m}^{3}$ 
which has a similar interpretation as (17) for the global ocean model. The time evolution of these three terms is shown in Fig. 12d for the case $t_{m}=23 \mathrm{yr}$, both for the system residing in the single (black curves) and in the multiple equilibrium regime (gray curves). Similar to the results in the global ocean model, the term $\bar{m}\left(\tilde{S}_{4}-\tilde{S}_{1}\right)$ is stabilizing (it leads to an increase in Atlantic salinity) and the term $\tilde{m}\left(\tilde{S}_{4}-\tilde{S}_{1}\right)$ is destabilizing. The two terms $\bar{m}\left(\tilde{S}_{4}-\tilde{S}_{1}\right)$ and $\tilde{m}\left(\tilde{S}_{4}-\tilde{S}_{1}\right)$ are very similar in both ME and $\mathrm{SE}$ regimes.

The change from $\mathrm{SE}$ to $\mathrm{ME}$ regime is, just as in the global ocean model, also related to a sign change in the term $\tilde{m}\left(\bar{S}_{4}-\bar{S}_{1}\right)$. The nice element in the box model is that the steady-state salinity contrast is given by

$$
\bar{S}_{4}-\bar{S}_{1}=-\frac{S_{0} F_{1}}{\bar{m}},
$$

and hence its sign is directly coupled to that of $F_{1}$. Because $\bar{m}>0$ and $\tilde{m}<0$ in both regimes, the term $\tilde{m}\left(\bar{S}_{4}-\bar{S}_{1}\right)$ will switch sign when $F_{1}$ (in the box model the equivalent to $M_{\mathrm{ov}}$ in the global model) switches sign, which is exactly at the boundary between SE and ME regime (Fig. 12b).

\section{Summary and discussion}

Using a fully implicit global ocean model coupled to an energy balance atmosphere model, we revisited the problem of the characterization of the multiple equilibrium (ME) regime of the Atlantic MOC through an indicator $\Sigma$ given by (1). Our ocean model certainly has many deficiencies (Dijkstra and Weijer 2005), such that western boundary currents are very broad, the winddriven gyre flows are relatively weak, and eddy processes are completely ignored. The discussion below should be considered with these limitations in mind. The main advantages of the model approach here is that 1) full bifurcation diagrams can be computed versus the freshwater flux parameter $\gamma_{p}$ and that 2) the freshwater balances over the Atlantic basin are accurately satisfied. A detailed connection can therefore be made between the position of the saddle-node bifurcations bounding the hysteresis regime of the Atlantic MOC and changes in the Atlantic freshwater budget.

Our aim was to provide a better physical picture of why the indicator $\Sigma$ has a zero on the on branch for the value of $\gamma_{L_{-}}$, where we find the saddle-node bifurcation $L_{-}$on the off branch. This is a nonlinear problem because entering the $\mathrm{ME}$ regime from the $\mathrm{SE}$ regime when $\gamma_{p}$ is increased is related to a change in the development of finite amplitude perturbations. The on state is stable to small perturbations in both SE and ME regime. Our approach was to add controlled perturbations (an anomalous freshwater flux) for a time $t_{m}$ and then analyze the differences in the evolution of the Atlantic freshwater budget between both ME and SE regimes.

Equation (17) clearly illustrates the importance of the freshwater fluxes at the southern boundary. Interactions between the perturbed flow and the background steady state, as well as nonlinear-driven freshwater transport, directly contribute to the tendency of the volumeaveraged salinity perturbation in the Atlantic basin. At the value of $\gamma_{p}$ where the steady-state value of $\Sigma \approx$ $M_{\text {ov }}\left(35^{\circ} \mathrm{S}\right)$ changes sign, the anomalous salinity transport associated with the $\tilde{v} \bar{S}$ term also changes sign. As the steady-state meridional velocity $\bar{v}$ does not change much with $\gamma_{p}$ on the on branch, the sign of $\Sigma$ is closely coupled to that of the steady-state salinity field at $35^{\circ} \mathrm{S}$. With increasing $\gamma_{p}$, the salinity at depth decreases (there is freshwater export in the ME regime) and hence the $\tilde{v} \bar{S}$ term becomes more destabilizing (again because $\tilde{v}$ is very similar for the $\mathrm{SE}$ and $\mathrm{ME}$ regime).

This connection between properties of the steady states and the processes controlling the evolution of perturbations was most clearly illustrated with the boxmodel analysis in section $5 \mathrm{c}$. When the MOC decreases because of the change in freshwater flux in the northern box, the interaction of the velocity perturbation and the steady-state salinity field transports salt into the Atlantic basin in the SE regime and freshwater in the ME regime. While in the box model the meridional advection terms in the salinity equation are the only relevant process, we have assumed that these terms are dominant in the global ocean model [as reflected in the Eq. (17)].

Climate models that were integrated as part of the SRES A1B scenario of the IPCC do not show any sign of abrupt change in the Atlantic MOC (Schmittner et al. 2005), although this does not prove that the models do not have a ME regime. Model intercomparison studies show no systematic differences in THC behavior and climate response between EMICs and atmosphereocean GCMs (CGCMs) (Gregory et al. 2005; Stouffer et al. 2006). The ME regime is present in simple coupled models (De Vries and Weber 2005; Rahmstorf et al. 2005; Weber et al. 2007) and in some more complex coupled models (Manabe and Stouffer 1988). Atmospheric feedbacks may indeed change the crucial role of the salt-advection feedback by affecting the east-west salinity difference at $35^{\circ} \mathrm{S}$ in response to a temporary decrease in the Atlantic MOC. However, when they would completely remove the $\mathrm{ME}$ regime, it becomes more difficult to explain the paleoclimatic record (Clark et al. 2002). 
Before discussing the applicability of $\Sigma$ as an indicator of multiple equilibria in GCMs and in observations, we mention explicitly that the model used here has a relatively large vertical diffusivity and hence the MOC is in the "mixing" regime. When $K_{V}$ is decreased, several cases can be distinguished: 1) the multiple equilibria disappear, 2) there are still multiple equilibria but because the MOC is more "wind driven" the quantity $\Sigma$ would not be a good indicator, and 3) the multiple equilibria remain and $\Sigma$ is still a good indicator. Support for 3 ) is provided by the results in Weber et al. (2007), but a detailed study of what happens with the bifurcation diagrams when decreasing $K_{V}$ would require much more work. It would also require a different model, as low vertical diffusivity regimes cannot be reached with the model used in this paper.

Calculating $\Sigma$ to investigate multistability in coupled GCMs is rather straightforward and has the advantage of inferring the stability regime from the equilibrium solution, without having to perform hosing experiments. When the MOC changes in these models, the freshwater flux field changes in a complicated way and there are also changes in the wind field and the heat flux field. However, based on the increased knowledge of the physics behind the indicator $\Sigma$, we think that it is a relevant diagnostic for the stability properties of the MOC in coupled GCMs as it is related to (intrinsic) advective processes in the Atlantic freshwater budget.

Yin and Stouffer (2007) compared the development of the MOC to freshwater perturbations in two different versions of the Geophysical Fluid Dynamics Laboratory (GFDL) model. They attributed the different behavior of the MOC in these models to the different character of the off states and to different atmospheric feedbacks. However, from their analysis it can be deduced that $\Sigma$ is negative in the model where the off state appears to be stable and is characterized by a reversed MOC. In the other model, $\Sigma$ is positive and a weakened on state is found, which evolves back to the on-state MOC when the hosing stops, regardless of the atmospheric feedbacks. This behavior is consistent with the hypothesis that the sign of $\Sigma$ determines the regime of the MOC.

It is interesting to see that the result on $\Sigma$ strongly depends on the depth of the zero contour of the MOC. When the position of the zero contour is at a shallower depth, $\Sigma$ tends to be more negative and hence there is a tendency towards the multiple equilibrium regime. This is actually seen in GCMs where the MOC shoals under increasing greenhouse gas concentrations (Stouffer et al. 2006). Also, in simulations of glacial climates, there are indications for a shoaling of the MOC (Weber et al. 2007), again pointing to a MOC that is more likely to be in a multiple equilibrium regime.
To address the important question of where the real ocean resides, estimates of $\Sigma$ can be made. Using data from an inversion of the World Ocean Circulation Experiment (WOCE) data by Holfort (1994), Weijer et al. (1999) concluded that the MOC exports freshwater at $30^{\circ} \mathrm{S}$. They determined a present-day value of $\Sigma \approx$ $M_{\mathrm{ov}}\left(30^{\circ} \mathrm{S}\right) \approx-0.3 \mathrm{~Sv}$ and no error estimate was given. Using a recent dataset (Gouretski and Koltermann 2004), we determined the zonally averaged salinity profile at $35^{\circ} \mathrm{S}$ (Fig. 13a) and calculated a zonal-mean velocity profile based on thermal wind balance with the same method as was used to obtain Fig. 8.2 in Van Aken (2007). An Ekman transport of $4 \mathrm{~Sv}$ was added to obtain a zero integral of the section-averaged volume transport (as required in steady state); the result is shown in Fig. 13b. From these profiles, we obtain $\Sigma=M_{\mathrm{ov}}\left(35^{\circ} \mathrm{S}\right) \approx-0.1 \mathrm{~Sv}$; an Ekman transport change of $2 \mathrm{~Sv}$ gives a difference of $0.02 \mathrm{~Sv}$ in $\Sigma$. Similar to the results in Weijer et al. (1999), this would indicate that the present-day MOC is in the ME regime. Obviously, this result probably has a large error bar because $\Sigma$ will be a highly fluctuating quantity affected by many processes not considered here (such as the effect of the Bering Strait transport). We hope, however, that this value for $\Sigma$ and the results in this paper will stimulate analysis of combined datasets from observations and model simulations (such as in Garzoli and Baringer 2007) to provide better estimates of presentday values of $\Sigma$.

The main result of this paper is that we provide a physical justification that a negative sign of $\Sigma$ is a good indicator for the multiple equilibrium regime of the MOC. The results in section $4 \mathrm{~b}$ show that when a freshwater perturbation is imposed on the on state of the MOC, $\Sigma$ is a measure for the anomalous freshwater transport into (out of) the Atlantic, induced by velocity perturbations and the background salinity field. This transport is stabilizing the MOC (making the Atlantic saltier) in the SE regime for which the background MOC is exporting salt. However, it is destabilizing (freshening the Atlantic) in case the MOC exports freshwater. The analysis fully supports and corroborates earlier descriptive explanations (Rahmstorf 1996; De Vries and Weber 2005; Dijkstra 2007) and provides the details of the processes involved.

Acknowledgments. The computations were done on the Huygens IBM p6 supercomputer at SARA Amsterdam. Use of these computing facilities was sponsored by the National Computing Facilities Foundation (NCF) under the project SH084-08 with financial support from the Netherlands Organization for Scientific Research (NWO). This work was supported by a NWO Toptalent Grant to one of the authors (MdT). We thank both Stefan 

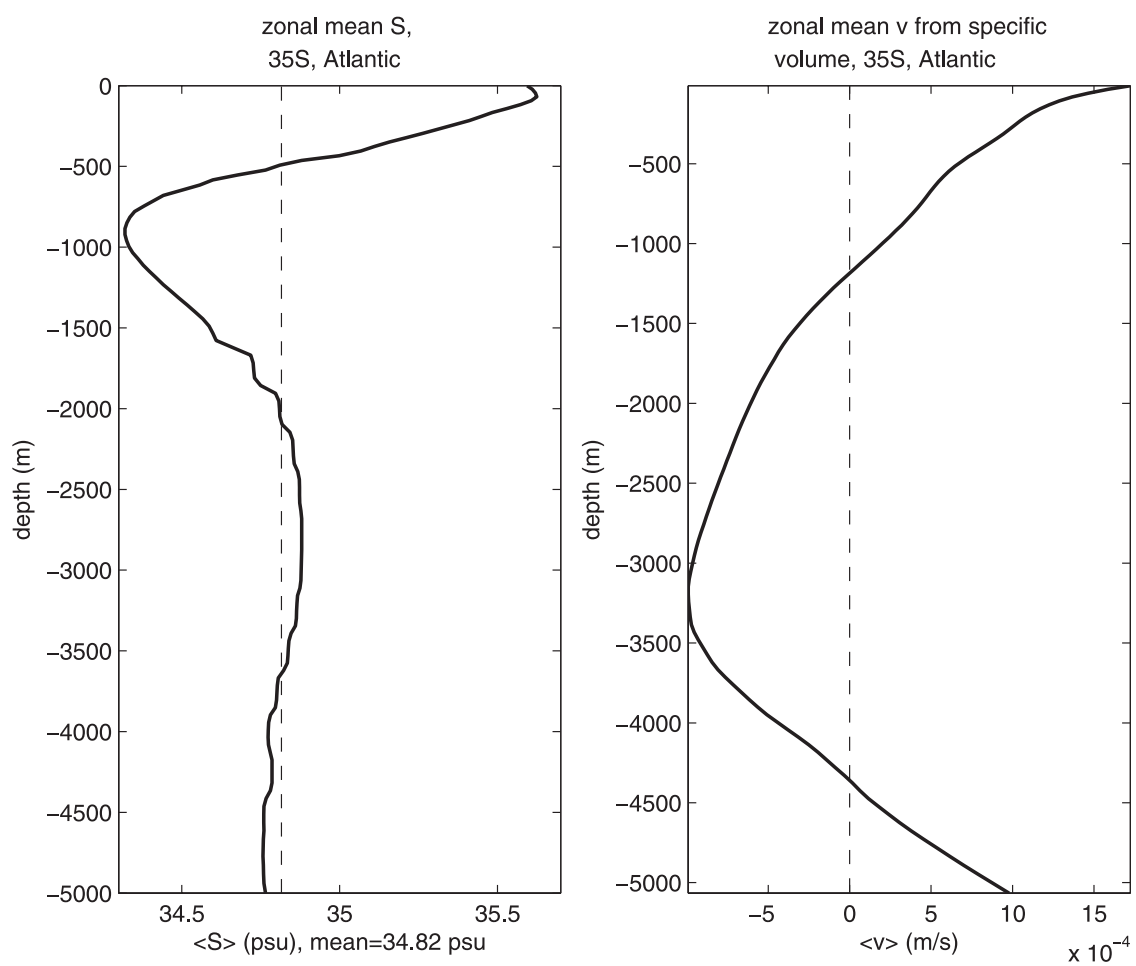

FIG. 13. (a) Zonally averaged salinity field from the dataset in Gouretski and Koltermann

(2004). (b) Zonally averaged meridional velocity field as determined from the same dataset.

Rahmstorf and another anonymous referee for very useful comments on the first version of this paper.

\section{REFERENCES}

Clark, P. U., N. G. Pisias, T. F. Stocker, and A. J. Weaver, 2002: The role of the thermohaline circulation in abrupt climate change. Nature, 415, 863-869.

De Vries, P., and S. L. Weber, 2005: The Atlantic freshwater budget as a diagnostic for the existence of a stable shut down of the meridional overturning circulation. Geophys. Res. Lett., 32, L09606, doi:10.1029/2004GL021450.

Dijkstra, H. A., 2007: Characterization of the multiple equilibria regime in a global ocean model. Tellus, 59A, 695-705.

— turning oscillations in thermohaline-driven flows. J. Fluid Mech., 331, 169-198.

—_, and W. Weijer, 2005: Stability of the global ocean circulation: Basic bifurcation diagrams. J. Phys. Oceanogr., 35, 933-948.

— L. A. Te Raa, and W. Weijer, 2004: A systematic approach to determine thresholds of the ocean's thermohaline circulation. Tellus, 56A, 362-370.

Garzoli, S., and M. O. Baringer, 2007: Meridional heat transport determined with expendable bathythermographs. Part II: South Atlantic transport. Deep-Sea Res., 54, 1402-1420.

Gouretski, V. V., and K. P. Koltermann, 2004: WOCE Global Hydrographic Climatology, Berichte des Bundesambtes für Seeschifffahrt und Hydrographie. Tech. Rep. AlfredWegener-Institut für Polar- und Meeresforschung 35, 52 pp.
Gregory, J. M., and Coauthors, 2005: A model intercomparison of changes in the Atlantic thermohaline circulation in response to increasing atmospheric $\mathrm{CO}_{2}$ concentration. Geophys. Res. Lett., 32, L12703, doi:10.1029/2005GL023209.

Holfort, J., 1994: Grossräumige Zirkulation und meridionale transporte in Südatlantic. Ph.D. thesis, Institut for Meereskunde, Kiel, Germany, 96 pp.

Kuhlbrodt, T., A. Griesel, M. Montoya, A. Levermann, and S. Rahmstorf, 2007: On the driving processes of the Atlantic meridional overturning circulation. Rev. Geophys., 45, RG2001, doi:10.1029/2004RG000166.

Levitus, S., 1994: Temperature. Vol. 4, World Ocean Atlas 1994, NOAA Atlas NESDIS 4, 117 pp.

Manabe, S., and R. J. Stouffer, 1988: Two stable equilibria of a coupled ocean-atmosphere model. J. Climate, 1, 841-866.

Rahmstorf, S., 1996: On the freshwater forcing and transport of the Atlantic thermohaline circulation. Climate Dyn., 12, 799-811.

- 2002: Ocean circulation and climate changes during the past 120000 years. Nature, 419, 207-214.

_ 2003: The current climate. Nature, 421, 699, doi:10.1038/ 421699a.

— - and Coauthors, 2005: Thermohaline circulation hysteresis: A model intercomparison. Geophys. Res. Lett., 32, L23605, doi:10.1029/2005GL023655.

Schmittner, A., M. Latif, and B. Schneider, 2005: Model projections of the North Atlantic thermohaline circulation for the 21 st century assessed by observations. Geophys. Res. Lett., 32, L23710, doi:10.1029/2005GL024368.

Stommel, H., 1961: Thermohaline convection with two stable regimes of flow. Tellus, 2, 244-230. 
Stouffer, R. J., and Coauthors, 2006: Investigating the causes of the response of the thermohaline circulation to past and future climate changes. J. Climate, 19, 1365-1387.

Trenberth, K. E., J. G. Olson, and W. G. Large, 1989: A global ocean wind stress climatology based on ECMWF analyses. NCAR Tech. Rep. NCARITN-338+STR, 93 pp.

Van Aken, H. M., 2007: The Oceanic Thermohaline Circulation. Springer, $326 \mathrm{pp}$.

Vellinga, M., R. A. Wood, and J. M. Gregory, 2002: Processes governing the recovery of a perturbed thermohaline circulation in HadCM3. J. Climate, 15, 764-780.

Walin, G., 1985: The thermohaline circulation and the control of ice ages. Paleogeogr. Paleoclimatol. Paleoecol., 50, 323-332.
Weber, S. L., and Coauthors, 2007: The modern and glacial overturning circulation in the Atlantic ocean in PMIP coupled model simulations. Climate Past, 3, 51-64.

Weijer, W., W. P. M. De Ruijter, H. A. Dijkstra, and P. J. Van Leeuwen, 1999: Impact of interbasin exchange on the Atlantic overturning circulation. J. Phys. Oceanogr., 29, 22662284.

Wunsch, C., 2002: What is the thermohaline circulation? Science, 298, 1179-1180.

Yin, J., and R. J. Stouffer, 2007: Comparison of the stability of the Atlantic thermohaline circulation in two coupled atmosphere-ocean general circulation models. J. Climate, 20, 4293-4315. 